\title{
$S O(N)$ Reformulated Link Invariants from Topological Strings
}

\author{
Pravina Borhade ${ }^{1}$, P. Ramadevi ${ }^{2}$ \\ Department of Physics, \\ Indian Institute of Technology Bombay, \\ Mumbai 400 076, India
}

\begin{abstract}
Large $N$ duality conjecture between $U(N)$ Chern-Simons gauge theory on $S^{3}$ and $A$-model topological string theory on the resolved conifold was verified at the level of partition function and Wilson loop observables. As a consequence, the conjectured form for the expectation value of the topological operators in $A$-model string theory led to a reformulation of link invariants in $U(N)$ Chern-Simons theory giving new polynomial invariants whose integer coefficients could be given a topological meaning. We show that the $A$-model topological operator involving $S O(N)$ holonomy leads to a reformulation of link invariants in $S O(N)$ Chern-Simons theory. Surprisingly, the $S O(N)$ reformulated invariants also has a similar form with integer coefficients. The topological meaning of the integer coefficients needs to be explored from the duality conjecture relating $S O(N)$ Chern-Simons theory to $A$-model closed string theory on orientifold of the resolved conifold background.
\end{abstract}

\footnotetext{
${ }^{1}$ E-mail: pravina@phy.iitb.ac.in

${ }^{2}$ Email: ramadevi@phy.iitb.ac.in
} 


\section{Introduction}

Within the last one decade we have seen interesting developments in the open string and closed string dualities. One such open-closed string duality conjecture relates $A$-model open topological string theory on the deformed conifold, equivalent to Chern-Simons gauge theory on $S^{3}[1]$, to the closed string theory on a resolved conifold.

Gopakumar-Vafa [2-4] showed that the free-energy expansion of $U(N)$ Chern-Simons field theory on $S^{3}$ at large $N$ resembles $A$-model topological string theory amplitudes on the resolved conifold. The conjecture was further tested at the level of observables in Chern-Simons theory. Ooguri-Vafa [5] considered the expectation value of a topological operator corresponding to a simple circle (called unknot) in submanifold $S^{3}$ of the deformed conifold and showed its form in the resolved conifold background. The results led to a new conjecture (usually referred to as Ooguri-Vafa conjecture) on the form for the expectation value of the topological operator for any knot or link in $S^{3}$.

Using group theory, Labastida-Marino [6] showed that the expectation value of the topological operators can be rewritten in terms of link invariants in $U(N)$ Chern-Simons field theory on $S^{3}$. This enabled verification of Ooguri-Vafa conjecture for many non-trivial knots [6-9]. Conversely, the Ooguri-Vafa conjecture led to a reformulation of Chern-Simons field theory invariants for links giving new polynomial invariants. The integer coefficients of these new polynomial invariants have topological meaning accounting for BPS states in the string theory. The challenge still remains in obtaining such integers within topological string theory.

Similar duality conjectures have been attempted between Chern-Simons gauge theories on three-manifolds other than $S^{3}$ and closed string theories. In ref. [10], $U(N)$ Chern-Simons free-energy expansion at large $N$ for many three-manifolds were derived and the expansion resembled partition function of a closed string theory on a Calabi-Yau background with one

kahler parameter. Unfortunately, the Chern-Simons free-energy expansion for other threemanifolds are not equivalent to the 't Hooft large $N$ perturbative expansion around a classical solution [11]. Hence we need to extract the perturbative expansion around a classical solution from the free-energy to obtain new duality conjectures.

For orbifolds of $S^{3}$, which gives Lens space $\mathcal{L}[p, 1] \equiv S^{3} / Z_{p}$, it is believed that the ChernSimons theory is dual to the $A$-model closed string theory on $A_{p-1}$ fibred over $P^{1}$ Calabi-Yau background. It was Marino [12] who showed that the perturbative Chern-Simons theory on Lens space $\mathcal{L}[p, 1]$ can be given a matrix model description. Also, hermitian matrix model description of $B$-model topological strings [13] was shown to be equivalent to Marino's matrix model using mirror symmetry [14]. It is still a challenging open problem to look for dual closed string description corresponding to $U(N)$ Chern-Simons theory on other three-manifolds. 
The extension of these duality conjectures for other gauge groups like $S O(N)$ and $S p(N)$ have also been studied. In particular, the free-energy expansion of the Chern-Simons theory based on $S O / S p$ gauge group was shown to be dual to $A$-model closed string theory on a orientifold of the resolved conifold background [15]. Further, using the topological vertex as a tool, Bouchard et al $[16,17]$ have determined unoriented closed string amplitude and unoriented open topological string amplitudes for a few orientifold toric geometry with or without $D$ branes.

It will be interesting to generalise Ooguri-Vafa conjecture by looking at the topological operator involving $S O / S p$ holonomy instead of the $U(N)$ holonomy. In this paper, we obtain new reformulated polynomial invariants in terms of the framed link polynomials in $S O(N)$ ChernSimons theory. Similar to the $U(N)$ result, the coefficients are indeed integers and the topological meaning in terms of the BPS invariants in string theory needs to be explored. Further, the reformulated invariant for knots in standard framing obeys the conjecture of BouchardFlorea-Marino [17] giving the integer coefficients corresponding to cross-cap $c=1$ unoriented open-string amplitude. We generalise the conjecture for any $r$-component framed links and have verified for few examples of framed knots and two-component framed links.

The organisation of the paper is as follows. In section 2, we present framed link invariants in $S O(N)$ Chern-Simons theory. In section 3, first we recapitulate the topological operator carrying $U(N)$ holonomy and then elaborate its generalization to $S O(N)$ holonomy. Section 4 contains some explicit results of the reformulated polynomial invariants. We present the integer coefficients in the cross-cap $c=1$ unoriented string amplitudes for few framed knots and links in section 5. In the concluding section 6 , we summarize the results obtained and pose open questions for future research. In appendix A, we present $S O(N)$ polynomials for few framed knots and framed links for some representations. In appendix B, the reformulated polynomial invariants for few non-trivial framed knots and framed links are presented.

\section{$2 S O(N)$ Chern-Simons Gauge theory and Framed Link invariants}

Chern-Simons gauge theory on $S^{3}$ based on the gauge group $S O(N)$ is described by the following action:

$$
S=\frac{k}{4 \pi} \int_{S^{3}} \operatorname{Tr}\left(A \wedge d A+\frac{2}{3} A \wedge A \wedge A\right)
$$


where $A$ is a gauge connection for gauge group $S O(N)$ and $k$ is the coupling constant. The observables in this theory are Wilson loop operators:

$$
W_{R_{1}, R_{2}, \ldots R_{r}}[L]=\prod_{i=1}^{r} \operatorname{Tr}_{R_{i}} U\left[\mathcal{K}_{i}\right]
$$

where $U\left[\mathcal{K}_{i}\right]=P\left[\exp \oint_{\mathcal{K}_{i}} A\right]$ denotes the holonomy of the $S O(N)$ gauge field $A$ around the component knot $\mathcal{K}_{i}$ of a $r$-component link $L$ carrying representation $R_{i}$. The expectation value of these Wilson loop operators are the $S O(N)$ link invariants:

$$
V_{\Lambda_{R_{1}}, \Lambda_{R_{2}}, \ldots \Lambda_{R_{r}}}[L](q, \lambda)=\left\langle W_{R_{1}, R_{2}, \ldots R_{r}}[L]\right\rangle(q, \lambda)=\frac{\int[\mathcal{D} A] e^{i S} W_{R_{1}, R_{2}, \ldots, R_{r}}[L]}{\int[\mathcal{D} A] e^{i S}},
$$

where $\Lambda_{R_{i}}$ 's denote the highest weights of the representation $R_{i}$ 's. The $S O(N)$ link invariants are polynomials in two variables

$$
q=\exp \left(\frac{2 \pi i}{k+N-2}\right), \lambda=q^{N-1}
$$

involving the coupling constant $k$ and the rank of the gauge group. These link invariants can be computed using the following two inputs:

(i) Any link can be drawn as a closure or plat of braids, (ii) The connection between ChernSimons theory and the Wess-Zumino conformal field theory.

The invariant for the unknot is equal to the quantum dimension of the representation $R$ living on the unknot:

$$
V_{\Lambda_{R}}[U](q, \lambda)=\operatorname{dim}_{q} R
$$

where the quantum dimension of the representation $R$ with highest weight $\Lambda_{R}$ is given by

$$
\operatorname{dim}_{q} R=\Pi_{\alpha>0} \frac{\left[\alpha \cdot\left(\rho+\Lambda_{R}\right)\right]}{[\alpha \cdot \rho]}
$$

where $\alpha$ 's are the positive roots and $\rho$ is the Weyl vector equal to the sum of the fundamental weights of the group $S O(N)$. The square bracket refers to the quantum number defined by

$$
[x]=\frac{\left(q^{x / 2}-q^{-x / 2}\right)}{\left(q^{1 / 2}-q^{-1 / 2}\right)}
$$

We shall now present the polynomials for various framed knots and links. For the unknot $U$ with an arbitrary framing $p$, carrying a representation $R$ of $S O(N)$, the polynomial is

$$
V_{\Lambda_{R}}\left[0^{(p)}\right](q, \lambda)=(-1)^{\ell p} q^{\left(p C_{R}\right)} V_{\Lambda_{R}}[U]
$$


where $\ell$ refers to the total number of boxes in the Young-Tableau of the representation $R$ and the quadratic Casimir $C_{R}=\frac{\left(\Lambda_{R}+2 \rho\right) \cdot \Lambda_{R}}{2}$ in terms of Young-Tableau is given by

$$
C_{R}=\frac{1}{2}\left((N-1) \ell+\ell+\sum_{i}\left(l_{i}^{2}-2 i l_{i}\right)\right) .
$$

Here $l_{i}$ denotes the number of boxes in the $i$-th row of the Young-Tableau of the representation R. Eqns.(A.1-A.11) in appendix A contain explicit $p$-framed unknot polynomials for few representations.

Now, we can write the $S O(N)$ framed knot invariants for torus knots of the type $(2,2 m+1)$ with framing $[p-(2 m+1)]$ as follows:

$$
V_{\Lambda_{R}}[K](q, \lambda)=(-1)^{\ell[p-(2 m-1)]} q^{p C_{R}} \sum_{R_{s} \in R \otimes R} \operatorname{dim}_{q} R_{s}(-1)^{\epsilon_{s}}\left(q^{C_{R}-C_{R_{s}} / 2}\right)^{2 m+1}
$$

where $\epsilon_{s}= \pm 1$ depending upon whether the representation $R_{s}$ appears symmetrically or antisymmetrically with respect to the tensor product $R \otimes R$ in the $S O(N)_{k}$ Wess-Zumino Witten model. Explicit polynomial expression for $p$ framed trefoil for some representations are presented in appendix A.

Similarly, $S O(N)$ invariants for framed torus links of the type $(2,2 m)$ can also be written. For example, the $S O(N)$ invariant for a Hopf link with linking number -1 and framing numbers $p_{1}$ and $p_{2}$ on the component knots carrying representations $R_{1}$ and $R_{2}$ will be

$$
V_{\Lambda_{R_{1}}, \Lambda_{R_{2}}}\left[H^{*}\left(p_{1}, p_{2}\right)\right](q, \lambda)=(-1)^{\ell_{1} p_{1}+\ell_{2} p_{2}} q^{p_{1} C_{R_{1}}+p_{2} C_{R_{2}}} \sum_{R_{s} \in R_{1} \otimes R_{2}} \operatorname{dim}_{q} R_{s} q^{C_{R_{1}}+C_{R_{2}}-C_{R_{s}}}
$$

We have presented the explicit framed Hopf link polynomials for some representations in the appendix A. Using the framed torus knot/link invariants, we can write the $S O(N)$ invariants for connected sums. For example, knot $K=K_{1} \# K_{2}$, where $K_{1}$ and $K_{2}$ are framed torus knots,

$$
V_{\Lambda_{R_{1}}}\left[K=K_{1} \# K_{2}\right](q, \lambda)=\frac{1}{V_{\Lambda_{R_{1}}}[U](q, \lambda)}\left(V_{\Lambda_{R_{1}}}\left[K_{1}\right](q, \lambda) V_{\Lambda_{R_{1}}}\left[K_{2}\right](q, \lambda)\right) .
$$

We can also consider a link $L$ obtained as a connected sum of a torus knot $K_{1}$ and a torus link $L_{1}$. The link invariant will be

$$
V_{\Lambda_{R_{1}, R_{2}}}\left[L=K_{1} \# L_{1}\right](q, \lambda)=\frac{1}{V_{\Lambda_{R_{1}}}[U](q, \lambda)}\left(V_{\Lambda_{R_{1}}}\left[K_{1}\right](q, \lambda) V_{\Lambda_{R_{1}}, \Lambda_{R_{2}}}\left[L_{1}\right](q, \lambda)\right)
$$

In the following section, we will see the reformulation of $S O(N)$ invariants giving new polynomial invariants. 


\section{$3 \quad$ Reformulated Link Invariants}

We will briefly review the new polynomial invariants obtained as a reformulation of link invariants in $U(N)$ Chern-Simons theory. Then, we address the modified group theoretic equations for the $S O(N)$ group and show that they also give a similar reformulated invariants.

\section{1 $U(N)$ Reformulated Link Invariants}

Ooguri and Vafa showed that the Wilson loop operators in Chern-Simons theory correspond to certain observables in the topological string theory giving another piece of evidence for Gopakumar-Vafa duality conjecture. The operators in the open topological string theory which contains information about links is given by [5]

$$
Z\left(\left\{U_{\alpha}\right\},\left\{V_{\alpha}\right\}\right)=\exp \left[\sum_{\alpha=1}^{r} \sum_{d=1}^{\infty} \frac{1}{d} \operatorname{Tr} U_{\alpha}^{d} \operatorname{Tr} V_{\alpha}^{d}\right]
$$

where $U_{\alpha}$ is the holonomy of the gauge connection $A$ around the component knot $\mathcal{K}_{\alpha}$ carrying the fundamental representation in the $U(N)$ Chern-Simons theory on $S^{3}$, and $V_{\alpha}$ is the holonomy of a gauge field $\tilde{A}$ around the same component knot carrying the fundamental representation in the $U(M)$ Chern-Simons theory on a Lagrangian three-cycle which intersects $S^{3}$ along the curve $\mathcal{K}_{\alpha}$. The above operator can be equivalently represented as

$$
Z\left(\left\{U_{\alpha}\right\},\left\{V_{\alpha}\right\}\right)=1+\sum_{\left\{\vec{k}^{(\alpha)}\right\}} \prod_{\alpha=1}^{r} \frac{1}{z_{\vec{k}^{(\alpha)}}} \gamma_{\vec{k}^{(\alpha)}}\left(U_{\alpha}\right) \gamma_{\vec{k}^{(\alpha)}}\left(V_{\alpha}\right)
$$

where

$$
z_{\vec{k}^{(\alpha)}}=\prod_{j} k_{j}^{(\alpha)} ! j^{k_{j}^{(\alpha)}}, \gamma_{\vec{k}^{(\alpha)}}\left(U_{\alpha}\right)=\prod_{j=1}^{\infty}\left(\operatorname{Tr} U_{\alpha}^{j}\right)^{k_{j}^{(\alpha)}} .
$$

Here $\vec{k}^{(\alpha)}=\left(k_{1}^{(\alpha)}, k_{2}^{(\alpha)}, \ldots\right)$ with $\left|\vec{k}^{(\alpha)}\right|=\sum_{j} k_{j}^{(\alpha)}$ and the sum is over all the vectors $\vec{k}^{(\alpha)}$ such that $\sum_{\alpha=1}^{r}\left|\vec{k}^{(\alpha)}\right|>0$. Using the following group theoretic Frobenius equations,

$$
\begin{gathered}
\gamma_{k_{1}}\left(U_{1}\right) \ldots \gamma_{k_{r}}\left(U_{r}\right)=\sum_{R_{1}, \ldots R_{r}} \prod_{\alpha=1}^{r} \chi_{R_{\alpha}}\left(C\left(\vec{k}^{(\alpha)}\right)\right) \operatorname{Tr}_{R_{1}}\left(U_{1}\right) \ldots \operatorname{Tr}_{R_{r}}\left(U_{r}\right) \\
\left.\left.\sum_{\vec{k}} \frac{1}{z_{\vec{k}}} \chi_{R_{1}}(C(\vec{k}))\right) \chi_{R_{2}}(C(\vec{k}))\right)=\delta_{R_{1} R_{2}}
\end{gathered}
$$

where $\chi_{R_{\alpha}}\left(C\left(\vec{k}^{(\alpha)}\right)\right)$ 's are characters of the symmetry group $S_{\ell_{\alpha}}$ with $\ell_{\alpha}=\sum_{j} j k_{j}^{(\alpha)}$ and $C\left(\vec{k}^{(\alpha)}\right)$ are the conjugacy classes associated to $\vec{k}^{(\alpha)}$ 's (denoting $k_{j}^{(\alpha)}$ cycles of length $j$ ), the operator can be shown to be

$$
Z\left(\left\{U_{\alpha}\right\},\left\{V_{\alpha}\right\}\right)=\sum_{\left\{R_{\alpha}\right\}} \prod_{\alpha=1}^{r} \operatorname{Tr}_{R_{\alpha}}\left(U_{\alpha}\right) \operatorname{Tr}_{R_{\alpha}}\left(V_{\alpha}\right)
$$


Ooguri and Vafa have conjectured a specific form for the vacuum expectation value (vev) of the topological operators (3.1) for knots [5] invoking the large $N$ topological string duality. This result was further refined for links [8] which is generalisable for framed links [9] as follows

$$
\begin{aligned}
\left\langle Z\left(\left\{U_{\alpha}\right\},\left\{V_{\alpha}\right\}\right)\right\rangle_{A} & =\exp \left[\sum_{d=1}^{\infty} \sum_{\left\{R_{\alpha}\right\}} \frac{1}{d} f_{\left(R_{1}, \ldots R_{r}\right)}\left(q^{d}, \lambda^{d}\right) \prod_{\alpha=1}^{r} \operatorname{Tr}_{R_{\alpha}} V_{\alpha}^{d}\right], \\
f_{\left(R_{1}, R_{2}, \ldots R_{r}\right)}(q, \lambda) & =\sum_{Q, s} \frac{1}{\left(q^{1 / 2}-q^{-1 / 2}\right)} N_{\left(R_{1}, \ldots R_{r}\right), Q, s} q^{s} \lambda^{Q}
\end{aligned}
$$

where the suffix $A$ on the vev implies that the expectation value is obtained by integrating the $U(N)$ gauge fields $A$ 's on $S^{3}$. Further, for framed links $N_{\left(R_{1}, \ldots R_{r}\right), Q, s}$ are integers. In fact, $f_{R_{1}, R_{2}, \ldots R_{r}}(q, \lambda)$ are the $U(N)$ reformulated polynomial invariants involving $U(N)$ Chern-Simons link invariants.

The general formula for the reformulated polynomial invariant $f$ (3.8 ) in terms of $U(N)$

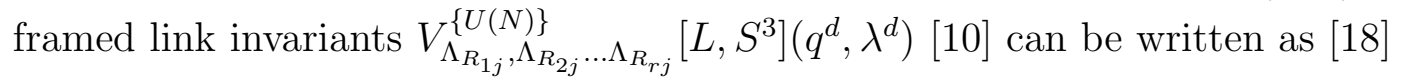

$$
\begin{aligned}
f_{R_{1}, R_{2}, \ldots R_{r}}(q, \lambda)= & \sum_{d, m=1}^{\infty}(-1)^{m-1} \frac{\mu(d)}{d m} \sum_{\left\{\vec{k}^{(\alpha j)}, R_{\alpha j}\right\}} \times \\
& \prod_{\alpha=1}^{r} \chi_{R_{\alpha}}\left(C\left(\left(\sum_{j=1}^{m} \vec{k}^{(\alpha j)}\right)_{d}\right)\right) \prod_{j=1}^{m} \frac{\left|C\left(\vec{k}^{(\alpha j)}\right)\right|}{\ell_{\alpha j} !} \times \\
& \chi_{R_{\alpha j}}\left(C\left(\vec{k}^{(\alpha j)}\right)\right) V_{\Lambda_{R_{1 j}}, \Lambda_{R_{2 j}} \ldots \Lambda_{R_{r j}}}\left[L, S^{3}\right]\left(q^{d}, \lambda^{d}\right)
\end{aligned}
$$

where $\mu(d)$ is the Moebius function defined as follows: if $d$ has a prime decomposition $\left(\left\{p_{i}\right\}\right)$, $d=\prod_{i=1}^{a} p_{i}^{m_{i}}$, then $\mu(d)=0$ if any of the $m_{i}$ is greater than one. If all $m_{i}=1$, then $\mu(d)=(-1)^{a}$. The second sum in the above equation runs over all vectors $\vec{k}^{(\alpha j)}$, with $\alpha=1, \ldots r$ and $j=1, \ldots m$, such that $\sum_{\alpha=1}^{r}\left|\vec{k}^{(\alpha j)}\right|>0$ for any $j$ and over representations $R_{\alpha j}$. Further $\vec{k}_{d}$ is defined as follows: $\left(\vec{k}_{d}\right)_{d i}=k_{i}$ and has zero entries for the other components. Therefore, if $\vec{k}=\left(k_{1}, k_{2}, \ldots\right)$, then

$$
\vec{k}_{d}=\left(0, \ldots, 0, k_{1}, 0, \ldots, 0, k_{2}, 0, \ldots\right),
$$

where $k_{1}$ is in the $d$-the entry, $k_{2}$ in the $2 d$-th entry, and so on. Hence, one can directly evaluate $f$ from $U(N)$ framed link invariants and verify the conjecture (3.8). Further refinement of eqn. (3.9) revealing the BPS structure has been presented in [8]:

$$
f_{R_{1}, R_{2}, \ldots R_{r}}(q, \lambda)=\sum_{R_{1}^{\prime}, \ldots R_{r}^{\prime}} M_{R_{1}, \ldots R_{r} ; R_{1}^{\prime}, \ldots R_{r}^{\prime}} \hat{f}_{\left(R_{1}^{\prime}, \ldots R_{r}^{\prime}\right)}(q, \lambda) .
$$

where

$$
M_{R_{1}, \ldots R_{r} ; R_{1}^{\prime}, \ldots R_{r}^{\prime}}=\sum_{R_{1}^{\prime \prime}, \ldots R_{r}^{\prime \prime}} \prod_{\alpha=1}^{r} C_{R_{\alpha} R_{\alpha}^{\prime} R_{\alpha}^{\prime \prime}} S_{R_{\alpha}^{\prime \prime}}(q)
$$




$$
\hat{f}_{\left(R_{1}^{\prime}, \ldots R_{r}^{\prime}\right)}(q, \lambda)=\left(q^{-1 / 2}-q^{1 / 2}\right)^{r-2} \sum_{g \geq 0, Q} \hat{N}_{\left(R_{1}^{\prime}, \ldots R_{r}^{\prime}\right), g, Q}\left(q^{-1 / 2}-q^{1 / 2}\right)^{2 g} \lambda^{Q},
$$

In eqn.(3.12) $, R_{\alpha}, R_{\alpha}^{\prime}, R_{\alpha}^{\prime \prime}$ are representations of the symmetric group $S_{\ell_{\alpha}}$ which can be labelled by a Young-Tableau with a total of $\ell_{\alpha}$ boxes and $C_{R R^{\prime} R^{\prime \prime}}$ are the Clebsch-Gordan coefficients of the symmetric group. $S_{R}(q)$ is non-zero only for hook representations. For such hook representation having $\ell-d$ boxes in the first row of the Young Tableau with total $\ell$ boxes, $S_{R}(q)=(-1)^{d} q^{-(\ell-1) / 2+d}$.

\section{2 $\mathrm{SO}(\mathrm{N})$ Reformulated Invariants}

As a problem within Chern-Simons field theory, we could take the same operator (3.1) to carry $S O(N)$ holonomy instead of $U(N)$ holonomy. We shall denote the topological operator involving $S O(N)$ holonomy as

$$
\tilde{Z}\left(\left\{\tilde{U}_{\alpha}\right\},\left\{\tilde{V}_{\alpha}\right\}\right)=\exp \left[\sum_{\alpha=1}^{r} \sum_{d=1}^{\infty} \frac{1}{d} \operatorname{Tr} \tilde{U}_{\alpha}^{d} \operatorname{Tr} \tilde{V}_{\alpha}^{d}\right]
$$

where $\tilde{U}_{\alpha}$ is the holonomy of the gauge connection $A$ around the component knot $\mathcal{K}_{\alpha}$ carrying the defining representation in the $S O(N)$ Chern-Simons theory on $S^{3}$, and $\tilde{V}_{\alpha}$ is the holonomy of a gauge field $\tilde{A}$ around the same component knot carrying the defining representation in the $S O(M)$ Chern-Simons theory on a Lagrangian three-cycle $\mathcal{C}$ which intersects $S^{3}$ along the curve $\mathcal{K}_{\alpha}$. In the context of the duality of the $S O$ Chern-Simons theory to the closed string on the orientifold of the resolved conifold [15], the gauge group of the Chern-Simons theory on $S^{3}$ alone has to be $S O$. Even if we choose $S O$ gauge group for Chern-Simons theory on $S^{3}$ and $S O$ Chern-Simons theory on Lagrangian cycle $\mathcal{C}$, the results relevant to open topological string amplitude [17] in the orientifold background will be unaltered.

Similar to eqn.(3.2), the above operator can be equivalently represented as

$$
\tilde{Z}\left(\left\{\tilde{U}_{\alpha}\right\},\left\{\tilde{V}_{\alpha}\right\}\right)=1+\sum_{\left\{\vec{k}^{(\alpha)}\right\}} \prod_{\alpha=1}^{r} \frac{1}{z_{\vec{k}^{(\alpha)}}} \gamma_{\vec{k}^{(\alpha)}}\left(\tilde{U}_{\alpha}\right) \gamma_{\vec{k}^{(\alpha)}}\left(\tilde{V}_{\alpha}\right)
$$

with the usual definitions for $z_{\vec{k}^{(\alpha)}}$ and $\gamma_{\vec{k}^{(\alpha)}}\left(U_{\alpha}\right)$ as given in eqn. (3.3).

Now, we need to modify the Frobenius equation which is one of the main results of the paper. The orthogonality relation eqn.(3.5) remains the same for the $S O$ case. The eqn.(3.4) will be modified as follows:

$$
\gamma_{k_{1}}\left(U_{1}\right) \ldots \gamma_{k_{r}}\left(U_{r}\right)=\sum_{R_{1}, \ldots R_{r}} \prod_{\alpha=1}^{r} \chi_{R_{\alpha}}\left(C\left(\vec{k}^{(\alpha)}\right)\right) \hat{\operatorname{Tr}}_{R_{1}}\left(U_{1}\right) \ldots \hat{\operatorname{Tr}}_{R_{r}}\left(U_{r}\right)
$$


where $\chi_{R_{\alpha}}\left(C\left(\vec{k}^{(\alpha)}\right)\right)$ 's are again characters of the symmetry group $S_{\ell_{\alpha}}$ with $\ell_{\alpha}=\sum_{j} j k_{j}^{(\alpha)}$ and $C\left(\vec{k}^{(\alpha)}\right)$ are the conjugacy classes associated to $\vec{k}^{(\alpha)}$ 's (denoting $k_{j}^{(\alpha)}$ cycles of length $j$ ). Notice that we have put a 'hat' in the trace function in the above equation. We shall explain the meaning of the 'hat' by presenting $\hat{\operatorname{Tr}}_{R}(U)$ for few $S O(N)$ representations (we denote representation $R$ by the highest weight $\Lambda_{R}$ for convenience):

$$
\begin{aligned}
\hat{T r}_{\lambda^{(1)}} U & =\operatorname{Tr}_{\lambda^{(1)}} U \\
\hat{\operatorname{Tr}} r_{2 \lambda^{(1)}} U & =\operatorname{Tr}_{2 \lambda^{(1)}} U+1 \\
\hat{\operatorname{Tr}}{\lambda^{(2)}} U & =\operatorname{Tr}_{\lambda^{(2)}} U \\
\hat{T} r_{3 \lambda^{(1)}} U & =\operatorname{Tr}_{3 \lambda^{(1)}} U+\operatorname{Tr}_{\lambda^{(1)}} U \\
\hat{T} r_{\lambda^{(1)}+\lambda^{(2)}} U & =\operatorname{Tr}_{\lambda^{(1)}+\lambda^{(2)}} U+\operatorname{Tr}_{\lambda^{(1)}} U \\
\hat{T} r_{\lambda^{(3)}} U & =\operatorname{Tr}_{\lambda^{(3)}} U \\
\hat{\operatorname{Tr}}{ }_{4 \lambda^{(1)}} U & =\operatorname{Tr}_{4 \lambda^{(1)}} U+\operatorname{Tr}_{2 \lambda^{(1)}} U+1 \\
\hat{T} r_{2 \lambda^{(1)}+\lambda^{(2)}} U & =\operatorname{Tr}_{2 \lambda^{(1)}+\lambda^{(2)}} U+\operatorname{Tr}_{2 \lambda^{(1)}} U+\operatorname{Tr}_{\lambda^{(2)}} U \\
\hat{T} r_{2 \lambda^{(2)}} U & =\operatorname{Tr}_{2 \lambda^{(2)}} U+\operatorname{Tr}_{2 \lambda^{(1)}} U+1 \\
\hat{T} r_{\lambda^{(1)}+\lambda^{(3)}} U & =\operatorname{Tr}_{\lambda^{(1)}+\lambda^{(3)}} U+\operatorname{Tr}_{\lambda^{(2)}} U \\
\hat{T} r_{\lambda^{(4)}} U & =\operatorname{Tr}_{\lambda^{(4)}} U .
\end{aligned}
$$

In principle, $\hat{T r}$ can be derived for arbitrary $S O(N)$ representation with highest weight $\Lambda_{R}=$ $\sum_{i=1} n_{i}^{(R)} \lambda^{(i)}$. In the next section, we will use the above set of $\hat{\operatorname{Tr}}$ (3.17) for obtaining explicit results on topological open-string amplitudes.

Using the eqns.(3.16, 3.5), it is not difficult to see that the $S 0$ topological operator (3.15) is equivalent to

$$
\tilde{Z}\left(\left\{\tilde{U}_{\alpha}\right\},\left\{\tilde{V}_{\alpha}\right\}\right)=\sum_{\left\{R_{\alpha}\right\}} \prod_{\alpha=1}^{r} \hat{\operatorname{Tr}}_{R_{\alpha}}\left(\tilde{U}_{\alpha}\right) \hat{\operatorname{Tr}}_{R_{\alpha}}\left(\tilde{V}_{\alpha}\right) .
$$

Similar to Ooguri-Vafa conjecture, we propose the following conjecture for the operator (3.14) involving $S O$ holonomy:

\section{Conjecture 1:}

$$
\begin{aligned}
e^{\mathcal{F}\left(\left\{\tilde{V}_{\alpha}\right\}\right)}=\left\langle\tilde{Z}\left(\left\{\tilde{U}_{\alpha}\right\},\left\{\tilde{V}_{\alpha}\right\}\right)\right\rangle_{A} & =\exp \left[\sum_{d=1}^{\infty} \sum_{\left\{R_{\alpha}\right\}} \frac{1}{d} g_{\left(R_{1}, \ldots R_{r}\right)}\left(q^{d}, \lambda^{d}\right) \prod_{\alpha=1}^{r} \operatorname{Tr}_{R_{\alpha}} \tilde{V}_{\alpha}^{d}\right] \\
g_{\left(R_{1}, R_{2}, \ldots R_{r}\right)}(q, \lambda) & =\sum_{Q, s} \frac{1}{\left(q^{1 / 2}-q^{-1 / 2}\right)} \tilde{N}_{\left(R_{1}, \ldots R_{r}\right), Q, s} q^{s} \lambda^{Q}
\end{aligned}
$$

where the suffix $A$ on the vev implies that the expectation value is obtained by integrating the $S O(N)$ gauge fields $A$ 's on $S^{3}$ and $\tilde{N}_{\left(R_{1}, \ldots R_{r}\right), Q, s}$ in eqn.(13.20) are integers. We have introduced 
$\mathcal{F}\left(\left\{\tilde{V}_{\alpha}\right\}\right)$ which we call as open-string partition function. Incidentally, $\mathcal{F}\left(\left\{\tilde{V}_{\alpha}\right\}\right)$ is a sum of oriented string partition function(untwisted sector) and unoriented string partition function (twisted sector) as presented in ref. [17].

The function $g_{R_{1}, R_{2} \ldots R_{r}}(q, \lambda)$ are the $S O(N)$ reformulated polynomial invariants involving framed link invariants in $S O(N)$ Chern-Simons theory. It is easy to see that the eqn.(3.9) can be accordingly modified for $S O$ group involving the expectation value of $\hat{\operatorname{Tr}} \tilde{U}_{R}(\underline{3.17})$ as follows:

$$
\begin{aligned}
g_{R_{1}, R_{2}, \ldots R_{r}}(q, \lambda)= & \sum_{d, m=1}^{\infty}(-1)^{m-1} \frac{\mu(d)}{d m} \sum_{\left\{\vec{k}^{(\alpha j)}, R_{\alpha j}\right\}} \times \\
& \prod_{\alpha=1}^{r} \chi_{R_{\alpha}}\left(C\left(\left(\sum_{j=1}^{m} \vec{k}^{(\alpha j)}\right)_{d}\right)\right) \prod_{j=1}^{m} \frac{\left|C\left(\vec{k}^{(\alpha j)}\right)\right|}{\ell_{\alpha j} !} \times \\
& \chi_{R_{\alpha j}}\left(C\left(\vec{k}^{(\alpha j)}\right)\right)\left\langle\prod_{\alpha=1}^{r} \hat{T r}_{R_{\alpha j}} \tilde{U}_{\alpha}\left[\mathcal{K}_{\alpha}\right]\right\rangle\left(q^{d}, \lambda^{d}\right)
\end{aligned}
$$

where the definitions of $\mu(d), \vec{k}^{(\alpha j)}$ and $\vec{k}_{d}$ are same as defined in the previous subsection. Substituting the $\hat{\operatorname{Tr}}$ (3.17) and rewriting in terms of $S O(N)$ framed link invariants (2.3), we have explicitly verified that the $S O(N)$ reformulated invariant obeys the conjectured eqn. (3.20) for many framed knots and framed links. This is one of the non-trivial results of the paper which we present in the next section and in appendix B.

Using eqn. (3.12), we can rewrite the $S O(N)$ reformulated polynomial invariants $g_{R_{1}, R_{2}, \ldots R_{r}}(q, \lambda)$ as

$$
g_{R_{1}, R_{2}, \ldots R_{r}}(q, \lambda)=\sum_{R_{1}^{\prime}, \ldots R_{r}^{\prime}} M_{R_{1}, \ldots R_{r} ; R_{1}^{\prime}, \ldots R_{r}^{\prime}} \hat{g}_{\left(R_{1}^{\prime}, \ldots R_{r}^{\prime}\right)}(q, \lambda)
$$

Unfortunately, $\hat{g}_{R_{1}, \ldots R_{r}}(q, \lambda)$ does not have a BPS structure like the one given in eqn.(3.13) for $U(N)$ holonomy. This has also been extensively studied in the works of Bouchard et al [17] where they conjecture an equation for $\hat{g}_{R}(q, \lambda)$ corresponding to knots in standard framing as follows:

$$
\frac{1}{2}\left(\hat{g}_{R}\left(q, \lambda^{\frac{1}{2}}\right)-(-1)^{\ell(R)} \hat{g}_{R}\left(q,-\lambda^{\frac{1}{2}}\right)\right)=\sum_{g, \beta} N_{R, g, \beta}^{c=1}\left(q^{\frac{1}{2}}-q^{-\frac{1}{2}}\right)^{2 g} \lambda^{\beta} .
$$

In this equation, $N_{R, g, \beta}^{c=1}$ are BPS invariants corresponding to unoriented open string amplitudes with one cross-cap. The above conjecture can be generalised for arbitrary $r$-component framed links $[L, \mathbf{p}]$ where $\mathbf{p}=\left(p_{1}, p_{2} \ldots p_{r}\right)$ denotes the framing numbers $p_{i}$ 's on the component knots $\mathcal{K}_{i}$ 's. For such $r$-component framed links $[L, \mathbf{p}]$, we propose the following conjecture:

\section{Conjecture 2}

$$
\frac{1}{2}\left(\hat{g}_{R_{1}, R_{2} \ldots R_{r}}\left(q, \lambda^{\frac{1}{2}}\right)-(-1)^{\sum_{\alpha=1}^{r} \ell\left(R_{\alpha}\right)\left(p_{\alpha}+1\right)} \hat{g}_{R_{1}, R_{2}, \ldots R_{r}}\left(q,-\lambda^{\frac{1}{2}}\right)\right)=
$$




$$
\sum_{g, \beta} N_{R_{1}, R_{2}, \ldots R_{r}, g, \beta}^{c=1}\left(q^{\frac{1}{2}}-q^{-\frac{1}{2}}\right)^{2 g+r-1} \lambda^{\beta}
$$

We have verified the above conjecture for many framed knots and framed two component links. In section 5, we have presented $N_{R_{1}, R_{2}, \ldots R_{r}, g, \beta}^{c=1}$ for some framed knots and framed two component links.

\section{Explicit Computation of $S O(N)$ reformulated invari- ants $g_{R_{1}, R_{2}, \ldots R_{r}}(q, \lambda)$}

In this section we compute the functions $g_{R_{1}, \ldots R_{r}}(q, \lambda)$ for various nontrivial framed knots and links and show that they obey the conjectured form (3.20). We shall denote the representations $R_{i}$ 's in $g_{R_{1}, \ldots R_{r}}(q, \lambda)$ by their highest weights $\Lambda_{R_{i}}$ 's.

- For unknot in standard framing, the reformulated invariant is non-zero only for the defining representation:

$$
V_{\lambda^{(1)}}[U](q, \lambda)=g_{\lambda^{(1)}}(q, \lambda)=\frac{1}{q-1}\left[q-1+q^{1 / 2}(-1+\lambda) \lambda^{-1 / 2}\right] .
$$

This simplifies the form for open-string partition function $\mathcal{F}\left(\left\{\tilde{V}_{\alpha}\right\}\right)$ in eqn. (3.19) as follows:

$$
\mathcal{F}(\tilde{V})=\sum_{d} \frac{1}{d}\left(1+\frac{\lambda^{d / 2}-\lambda^{-d / 2}}{q^{d / 2}-q^{-1 / 2}}\right) \operatorname{Tr} \tilde{V}^{d}
$$

- For unknot with arbitrary framing $p$

$$
\begin{aligned}
g_{\lambda^{(1)}}(q, \lambda)= & (-1)^{p} \lambda^{p / 2}\left(1+\frac{q^{1 / 2}(-1+\lambda)}{(-1+q) \lambda^{1 / 2}}\right) \\
g_{2 \lambda^{(1)}}(q, \lambda)= & \frac{1}{2(-1+q)^{2}(1+q)}\left[2(-1+q)^{2}(1+q)\right. \\
& +\lambda^{-1+p}\left(2 q^{p}(-1+\lambda)\left(-q+q^{1 / 2}\left(-1+q^{2}\right) \lambda^{1 / 2}+q^{2} \lambda\right)\right. \\
& +(-1)^{p}\left(-\left((-1)^{p}(1+q)\left(q^{1 / 2}+\lambda^{1 / 2}\right)^{2}\left(-1+q^{1 / 2} \lambda^{1 / 2}\right)^{2}\right)\right. \\
& -(-1+q)(q+\lambda)(-1+q \lambda))] \\
g_{\lambda^{(2)}}(q, \lambda)= & \frac{1}{2(-1+q)^{2}(1+q)}\left[\lambda ^ { - 1 + p } \left(2\left(q^{3 / 2}+\lambda^{1 / 2}\right)\left(-1+q^{1 / 2} \lambda^{1 / 2}\right)\right.\right. \\
& q^{1 / 2-p}(-1+\lambda)-(1+q)\left(q^{1 / 2}+\lambda^{1 / 2}\right)^{2}\left(-1+q^{1 / 2} \lambda^{1 / 2}\right)^{2} \\
& \left.\left.+(-1)^{p}(-1+q)(q+\lambda)(-1+q \lambda)\right)\right]
\end{aligned}
$$




$$
\begin{aligned}
& g_{3 \lambda^{(1)}}(q, \lambda)= \frac{1}{(-1+q)^{3}(1+q)\left(1+q+q^{2}\right)}\left[(-1)^{p}\left(-1+q^{p}\right)\left(q^{1 / 2}+\lambda^{1 / 2}\right)\right. \\
&\left(-1+q^{1 / 2} \lambda^{1 / 2}\right)(-1+\lambda) q^{1 / 2} \lambda^{3(-1+p) / 2}\left(\lambda^{1 / 2}-q^{3 / 2}(-1+\right. \\
&\left.q(-1+\lambda)+q^{5 / 2} \lambda^{1 / 2}+\lambda\right)+q^{p}\left(-q^{1 / 2}+q\left(-1+q^{2}\right) \lambda^{1 / 2}+\right. \\
&\left.\left.\left.q^{7 / 2} \lambda\right)+q^{2 p}\left(-q^{1 / 2}+q\left(-1+q^{2}\right) \lambda^{1 / 2}+q^{7 / 2} \lambda\right)\right)\right] \\
& g_{\lambda^{(1)}+\lambda^{(2)}}(q, \lambda)= \frac{-1}{(-1+q)^{3}(1+q)}\left[(-1)^{p} q^{1 / 2-p}\left(-1+q^{p}\right)\left(q^{1 / 2}+\lambda^{1 / 2}\right)\right. \\
&\left(-1+q^{1 / 2} \lambda^{1 / 2}\right)(-1+\lambda) \lambda^{3(-1+p) / 2}\left(q^{p}\left(q^{1 / 2}+\lambda^{1 / 2}\right)\right. \\
&\left.\left.\left(-1+q^{3 / 2} \lambda^{1 / 2}\right)+q^{3 / 2}+\lambda^{1 / 2}-q^{2} \lambda^{1 / 2}-q^{1 / 2} \lambda\right)\right] \\
& g_{\lambda^{(3)}}(q, \lambda)= \frac{1}{(-1+q)^{3}(1+q)\left(1+q+q^{2}\right)}\left[(-1)^{p} q^{1 / 2-3 p}\left(-1+q^{p}\right)\right. \\
&\left(q^{1 / 2}+\lambda^{1 / 2}\right)\left(-1+q^{1 / 2} \lambda^{1 / 2}\right)(-1+\lambda) \lambda^{3(-1+p) / 2} \\
&\left(q^{7 / 2}-q\left(-1+q^{2}\right) \lambda^{1 / 2}-q^{1 / 2} \lambda+q^{p}\left(q^{7 / 2}-\right.\right. \\
&\left.q\left(-1+q^{2}\right) \lambda^{1 / 2}-q^{1 / 2} \lambda\right)+q^{2 p}\left(-\left(q^{3 / 2}(1+q)\right)\right. \\
&\left.\left.\left.+\left(-1+q^{4}\right) \lambda^{1 / 2}+q^{3 / 2}(1+q) \lambda\right)\right)\right]
\end{aligned}
$$

Substituting values for $p$, the above equations reduce to the conjectured form (3.20).

1. For unknot with framing $p=1$, we get

$$
\begin{aligned}
g_{\lambda^{(1)}}(q, \lambda) & =\frac{-1}{q-1}\left[(q-1) \lambda^{1 / 2}+q^{1 / 2}(-1+\lambda)\right] \\
g_{2 \lambda^{(1)}}(q, \lambda) & =\frac{1}{q-1}\left[\left(-1+q^{1 / 2} \lambda^{1 / 2}\right)\left(1+q(-1+\lambda)+q^{1 / 2} \lambda^{3 / 2}\right)\right] \\
g_{\lambda^{(2)}}(q, \lambda) & =\frac{-1}{q-1}\left[\left(-q^{-1 / 2} \lambda^{1 / 2}+\lambda\right)\left(-1+q+q^{1 / 2} \lambda^{1 / 2}+\lambda\right)\right]
\end{aligned}
$$

2. For unknot with framing two

$$
\begin{aligned}
g_{\lambda^{(1)}}(q, \lambda)= & \frac{1}{q-1}\left[(q-1) \lambda+q^{1 / 2}(-1+\lambda) \lambda^{1 / 2}\right] \\
g_{2 \lambda^{(1)}}(q, \lambda)= & \frac{1}{q-1}\left[( - 1 + \lambda ) \left\{1-q+\lambda-2 q \lambda+q^{1 / 2} \times\right.\right. \\
& \left.\left.\left(-1+q^{2}\right) \lambda^{3 / 2}+q^{2} \lambda^{2}\right\}\right] \\
g_{\lambda^{(2)}}(q, \lambda)= & \frac{-1}{q-1}\left[q^{-3 / 2}\left(q^{3 / 2}+\lambda^{1 / 2}\right)\left(-1+q^{1 / 2} \lambda^{1 / 2}\right)(-1+\lambda) \lambda\right]
\end{aligned}
$$

- We have presented the reformulated invariants for few framed knots and two component links in appendix B. 


\section{$5 \quad N_{\left(R_{1}, \ldots R_{r}\right), g, Q}^{c=1}$ Computation}

We shall now compute the integer coefficients (3.24) corresponding to cross-cap $c=1$ unoriented open string amplitude obtained from $S O(N)$ reformulated invariants for various framed knots and framed links.

\subsection{Framed Knots}

1. For unknot with zero framing, the only non zero coefficient is $N_{\lambda^{(1)}, 0,0}^{c=1}=1$.

2. For unknot with framing $p=1$

$$
N_{\lambda^{(1)}, 0,1 / 2}^{c=1}=-1
$$

\begin{tabular}{|l|cc|}
\hline & $\beta=1 / 2$ & $3 / 2$ \\
\hline $\mathrm{g}=0$ & 1 & -1 \\
\hline \multicolumn{3}{|c}{$N_{\lambda^{(2)}, g, \beta}^{c=1}$}
\end{tabular}

\begin{tabular}{|c|cc|}
\hline & $\beta=3 / 2$ & $5 / 2$ \\
\hline $\mathrm{g}=0$ & 1 & -1 \\
\hline \multicolumn{3}{|c|}{$N_{\lambda^{(1)}+\lambda^{(2)}, g, \beta}^{c=1}$}
\end{tabular}

\begin{tabular}{|r|ccc|}
\hline & $\beta=1 / 2$ & $3 / 2$ & $5 / 2$ \\
\hline $\mathrm{g}=0$ & -1 & 4 & -3 \\
1 & 0 & 1 & -1 \\
\hline \multicolumn{3}{|c}{$N_{\lambda^{(3)}, g, \beta}^{c=1}$}
\end{tabular}

3. For unknot with framing $p=2$ :

$$
N_{\lambda^{(1)}, 0,1}^{c=1}=1
$$

$$
\begin{array}{|c|cc|}
\hline & \beta=3 / 2 & 5 / 2 \\
\hline \mathrm{g}=0 & 1 & -1 \\
\hline \multicolumn{3}{|c}{N_{2 \lambda^{(1)}, 0,3 / 2}^{c=1}}
\end{array}
$$

\begin{tabular}{|r|cc|}
\hline & $\beta=3 / 2$ & $5 / 2$ \\
\hline $\mathrm{g}=0$ & 3 & -3 \\
1 & 1 & -1 \\
\hline \multicolumn{3}{|c}{$N_{\lambda(2), g, \beta}^{c=1}$}
\end{tabular}

\begin{tabular}{|r|ccc|}
\hline & $\beta=2$ & 3 & 4 \\
\hline $\mathrm{g}=0$ & 1 & -4 & 3 \\
1 & 0 & -1 & 1 \\
\hline \multicolumn{3}{|c}{$N_{3 \lambda^{(1)}, g, \beta}^{c=1}$}
\end{tabular}




\begin{tabular}{|r|ccc|}
\hline & $\beta=2$ & 3 & 4 \\
\hline $\mathrm{g}=0$ & 9 & -28 & 19 \\
1 & 6 & -27 & 21 \\
2 & 1 & -9 & 8 \\
3 & 0 & -1 & 1 \\
\hline
\end{tabular}

\begin{tabular}{r|ccc|}
\hline & $\beta=2$ & 3 & 4 \\
\hline $\mathrm{g}=0$ & 13 & -36 & 23 \\
1 & 16 & -57 & 41 \\
2 & 7 & -36 & 29 \\
3 & 1 & -10 & 9 \\
4 & 0 & -1 & 1 \\
\multicolumn{4}{c}{$N_{\lambda^{(3)}, g, \beta}^{c=1}$}
\end{tabular}

4. For trefoil knot in standard framing, the results are agreeing with the tables given in Ref. [17].

5. For trefoil knot with framing $p=1$

\begin{tabular}{r|ccc|}
\hline & $\beta=3 / 2$ & $5 / 2$ & $7 / 2$ \\
\hline $\mathrm{g}=0$ & -3 & 3 & -1 \\
1 & -1 & 1 & 0 \\
\hline \multicolumn{3}{c}{$N_{\lambda^{(1)}, g, \beta}^{c=1}$}
\end{tabular}

\begin{tabular}{|r|ccccc|}
\hline & $\beta=5 / 2$ & $7 / 2$ & $9 / 2$ & $11 / 2$ & $13 / 2$ \\
\hline $\mathrm{g}=0$ & 16 & -69 & 111 & -79 & 21 \\
1 & 20 & -146 & 307 & -251 & 70 \\
2 & 8 & -128 & 366 & -330 & 84 \\
3 & 1 & -56 & 230 & -220 & 45 \\
4 & 0 & -12 & 79 & -78 & 11 \\
5 & 0 & -1 & 14 & -14 & 1 \\
6 & 0 & 0 & 1 & -1 & 0 \\
\hline
\end{tabular}

\begin{tabular}{|r|ccccc|}
\hline & $\beta=5 / 2$ & $7 / 2$ & $9 / 2$ & $11 / 2$ & $13 / 2$ \\
\hline $\mathrm{g}=0$ & 30 & -114 & 167 & -111 & 28 \\
1 & 55 & -311 & 587 & -457 & 126 \\
2 & 36 & -367 & 912 & -791 & 210 \\
3 & 10 & -230 & 770 & -715 & 165 \\
4 & 1 & -79 & 376 & -364 & 66 \\
5 & 0 & -14 & 106 & -105 & 13 \\
6 & 0 & -1 & 16 & -16 & 1 \\
7 & 0 & 0 & 1 & -1 & 0 \\
\hline
\end{tabular}




$$
N_{\lambda(2)}^{c=1}, g, \beta
$$

6. For trefoil knot with framing $p=2$

$$
\begin{array}{|r|ccc|}
\hline & \beta=2 & 3 & 4 \\
\hline \mathrm{g}=0 & 3 & -3 & 1 \\
1 & 1 & -1 & 0 \\
N_{\lambda(1)}^{c=1}, \beta
\end{array}
$$

\begin{tabular}{|r|rrrrr|}
\hline & $\beta=7 / 2$ & $9 / 2$ & $11 / 2$ & $13 / 2$ & $15 / 2$ \\
\hline $\mathrm{g}=0$ & 30 & -114 & 167 & -111 & 28 \\
1 & 55 & -311 & 587 & -457 & 126 \\
2 & 36 & -367 & 912 & -791 & 210 \\
3 & 10 & -230 & 770 & -715 & 165 \\
4 & 1 & -79 & 376 & -364 & 66 \\
5 & 0 & -14 & 106 & -105 & 13 \\
6 & 0 & -1 & 16 & -16 & 1 \\
7 & 0 & 0 & 1 & -1 & 0 \\
\hline \multicolumn{7}{c}{$N_{2 \lambda(1), g, \beta}^{c=1}$} \\
\end{tabular}

\begin{tabular}{|r|rrrrr|}
\hline & $\beta=7 / 2$ & $9 / 2$ & $11 / 2$ & $13 / 2$ & $15 / 2$ \\
\hline $\mathrm{g}=0$ & 50 & -174 & 237 & -149 & 36 \\
1 & 125 & -601 & 1042 & -776 & 210 \\
2 & 120 & -919 & 2046 & -1709 & 462 \\
3 & 55 & -771 & 2222 & -2001 & 495 \\
4 & 12 & -376 & 1443 & -1365 & 286 \\
5 & 1 & -106 & 574 & -560 & 91 \\
6 & 0 & -16 & 137 & -136 & 15 \\
7 & 0 & -1 & 18 & -18 & 1 \\
8 & 0 & 0 & 1 & -1 & 0 \\
\hline \multicolumn{7}{|c}{$N_{\lambda(2), g, \beta}^{c=1}$} \\
\end{tabular}

7. For connected sum Trefoil \# Trefoil with zero framing 


\begin{tabular}{r|cccc|}
\hline & $\beta=2$ & 3 & 4 & 5 \\
\hline $\mathrm{g}=0$ & 8 & -14 & 9 & -2 \\
1 & 6 & -11 & 6 & -1 \\
2 & 1 & -2 & 1 & 0 \\
\hline \multicolumn{5}{c}{$N_{\lambda^{(1)}, g, \beta}^{c=1}$}
\end{tabular}

\begin{tabular}{r|rrrrrrrr|}
\hline & $\beta=7 / 2$ & $9 / 2$ & $11 / 2$ & $13 / 2$ & $15 / 2$ & $17 / 2$ & $19 / 2$ & $21 / 2$ \\
\hline $\mathrm{g}=0$ & 143 & -831 & 1950 & -2366 & 1561 & -525 & 66 & 2 \\
1 & 404 & -3144 & 8854 & -11819 & 7544 & -1488 & -596 & 245 \\
2 & 464 & -5419 & 19211 & -28097 & 14046 & 6348 & -9194 & 2641 \\
3 & 277 & -5379 & 25184 & -40255 & 6296 & 44160 & -41756 & 11473 \\
4 & 90 & -3292 & 21666 & -38551 & -18588 & 110890 & -98450 & 26235 \\
5 & 15 & -1256 & 12654 & -26241 & -38613 & 159091 & -141400 & 35750 \\
6 & 1 & -290 & 5048 & -13093 & -36589 & 147270 & -133378 & 31031 \\
7 & 0 & -37 & 1352 & -4787 & -21053 & 92681 & -85919 & 17763 \\
8 & 0 & -2 & 232 & -1243 & -7860 & 40544 & -38455 & 6784 \\
9 & 0 & 0 & 23 & -215 & -1917 & 12353 & -11954 & 1710 \\
10 & 0 & 0 & 1 & -22 & -295 & 2574 & -2531 & 273 \\
11 & 0 & 0 & 0 & -1 & -26 & 350 & -348 & 25 \\
12 & 0 & 0 & 0 & 0 & -1 & 28 & -28 & 1 \\
13 & 0 & 0 & 0 & 0 & 0 & 1 & -1 & 0 \\
\hline
\end{tabular}




\begin{tabular}{r|rrrrrrrr|}
\hline $\mathrm{g}=0$ & $\beta=7 / 2$ & $9 / 2$ & $11 / 2$ & $13 / 2$ & $15 / 2$ & $17 / 2$ & $19 / 2$ & $21 / 2$ \\
1 & 227 & -1237 & 2756 & -3206 & 2045 & -671 & 84 & 2 \\
2 & 801 & -5621 & 14872 & -19187 & 12269 & -2861 & -564 & 291 \\
3 & 1190 & -11771 & 38341 & -54346 & 29773 & 5595 & -12485 & 3697 \\
4 & 955 & -14403 & 59796 & -92245 & 27489 & 67819 & -68353 & 18942 \\
5 & 444 & -11132 & 61614 & -104212 & -18925 & 211571 & -190697 & 51337 \\
6 & 119 & -5578 & 43750 & -83517 & -79482 & 364896 & -323843 & 83655 \\
7 & 17 & -1803 & 21761 & -49317 & -100043 & 404876 & -363462 & 87971 \\
8 & 1 & -362 & 7561 & -21758 & -73516 & 307780 & -281842 & 62136 \\
9 & 0 & -41 & 1795 & -7097 & -35330 & 165164 & -154547 & 30056 \\
10 & 0 & -2 & 277 & -1653 & -11446 & 63250 & -60402 & 9976 \\
11 & 0 & 0 & 25 & -258 & -2483 & 17202 & -16719 & 2233 \\
12 & 0 & 0 & 1 & -24 & -346 & 3248 & -3201 & 322 \\
13 & 0 & 0 & 0 & -1 & -28 & 405 & -403 & 27 \\
14 & 0 & 0 & 0 & 0 & -1 & 30 & -30 & 1 \\
& 0 & 0 & 0 & 0 & 0 & 1 & -1 & 0 \\
\hline
\end{tabular}

\subsection{Framed Links}

1. Hopf Link

We take Hopf Link $H\left(p_{1}, p_{2}\right)$ with linking number -1 and framing on the two component knots as $p_{1}$ and $p_{2}$. The integers $N_{\left(R_{1}, R_{2}\right), g, \beta}^{c=1}$ for various combinations of $p_{1}$ and $p_{2}$ are tabulated below.

$$
\begin{aligned}
& \underline{p_{1}=0=p_{2}} \\
& N_{\left(\lambda^{(1)}, \lambda^{(1)}\right), 0,1 / 2}^{c=1}=1 \\
& \begin{array}{|l|cc|}
\hline & \beta=-1 & 0 \\
\hline \mathrm{g}=0 & -1 & 1 \\
\hline \multicolumn{2}{|c|}{N_{\left(2 \lambda^{(1)}, \lambda^{(1)}\right), g, \beta}^{c=1}}
\end{array} \\
& \begin{array}{|l|cc|}
\hline & \beta=-3 / 2 & -1 / 2 \\
\hline \mathrm{g}=0 & -1 & 1 \\
\hline
\end{array} \\
& N_{\left(3 \lambda^{(1)}, \lambda^{(1)}\right), g, \beta}^{c=1} \\
& \begin{array}{|l|cc|}
\hline & \beta=-1 / 2 & 1 / 2 \\
\hline \mathrm{g}=0 & 1 & -1 \\
\hline
\end{array} \\
& N_{\left(\lambda^{(1)}+\lambda^{(2)}, \lambda^{(1)}\right), g, \beta}^{c=1}
\end{aligned}
$$


$\underline{p_{1}=1=p_{2}}$

\begin{tabular}{|l|cc|}
\hline & $\beta=1 / 2$ & $3 / 2$ \\
\hline $\mathrm{g}=0$ & -1 & 1 \\
\hline
\end{tabular}

$N_{\left(\lambda^{(1)}, \lambda^{(1)}\right), g, \beta}^{c=1}$

\begin{tabular}{|l|cc|}
\hline & $\beta=3 / 2$ & $5 / 2$ \\
\hline $\mathrm{g}=0$ & -1 & 1 \\
\hline
\end{tabular}

$N_{\left(2 \lambda^{(1)}, \lambda^{(1)}\right), g, \beta}^{c=1}$

\begin{tabular}{|l|cc|}
\hline & $\beta=5 / 2$ & $7 / 2$ \\
\hline $\mathrm{g}=0$ & -1 & 1 \\
\hline
\end{tabular}

$N_{\left(3 \lambda^{(1)}, \lambda^{(1)}\right), g, \beta}^{c=1}$

\begin{tabular}{|r|ccc|}
\hline & $\beta=3 / 2$ & $5 / 2$ & $7 / 2$ \\
\hline $\mathrm{g}=0$ & 6 & -19 & 13 \\
1 & 1 & -8 & 7 \\
2 & 0 & -1 & 1 \\
\hline
\end{tabular}

$N_{\left(\lambda^{(1)}+\lambda^{(2)}, \lambda^{(1)}\right), g, \beta}^{c=1}$

\begin{tabular}{|r|ccc|}
\hline & $\beta=1 / 2$ & $3 / 2$ & $5 / 2$ \\
\hline $\mathrm{g}=0$ & 1 & -5 & 4 \\
1 & 0 & -1 & 1 \\
\hline
\end{tabular}

$N_{\left(\lambda^{(2)}, \lambda^{(1)}\right), g, \beta}^{c=1}$

\begin{tabular}{|r|cccc|}
\hline & $\beta=1 / 2$ & $3 / 2$ & $5 / 2$ & $7 / 2$ \\
\hline $\mathrm{g}=0$ & -1 & 15 & -36 & 22 \\
1 & 0 & 7 & -29 & 22 \\
2 & 0 & 1 & -9 & 8 \\
3 & 0 & 0 & -1 & 1 \\
\hline \multicolumn{4}{c}{$N_{\left(\lambda^{(3)}, \lambda^{(1)}\right), g, \beta}^{c=1}$}
\end{tabular}

$\underline{p_{1}=2=p_{2}}$

\begin{tabular}{|l|cc|}
\hline & $\beta=3 / 2$ & $5 / 2$ \\
\hline $\mathrm{g}=0$ & -1 & 1 \\
\hline
\end{tabular}

$N_{\left(\lambda^{(1)}, \lambda^{(1)}\right), g, \beta}^{c=1}$

\begin{tabular}{|r|ccc|}
\hline & $\beta=2$ & 3 & 4 \\
\hline $\mathrm{g}=0$ & -1 & 5 & -4 \\
1 & 0 & 1 & -1 \\
\hline
\end{tabular}

$N_{\left(2 \lambda^{(1)}, \lambda^{(1)}\right), g, \beta}^{c=1}$

\begin{tabular}{r|ccc|}
\hline & $\beta=2$ & 3 & 4 \\
\hline $\mathrm{g}=0$ & -4 & 13 & -9 \\
1 & -1 & 7 & -6 \\
2 & 0 & 1 & -1 \\
\hline
\end{tabular}

\begin{tabular}{r|cccc|}
\hline & $\beta=5 / 2$ & $7 / 2$ & $9 / 2$ & $11 / 2$ \\
\hline $\mathrm{g}=0$ & -1 & 15 & -36 & 22 \\
1 & 0 & 7 & -29 & 22 \\
2 & 0 & 1 & -9 & 8 \\
3 & 0 & 0 & -1 & 1 \\
\hline \multicolumn{3}{c}{$N_{\left(3 \lambda^{(1)}, \lambda^{(1)}\right), g, \beta}^{c=1}$}
\end{tabular}




\begin{tabular}{|r|cccc|}
\hline & $\beta=5 / 2$ & $7 / 2$ & $9 / 2$ & $11 / 2$ \\
\hline $\mathrm{g}=0$ & -13 & 106 & -204 & 111 \\
1 & -7 & 118 & -319 & 208 \\
2 & -1 & 55 & -219 & 165 \\
3 & 0 & 12 & -78 & 66 \\
4 & 0 & 1 & -14 & 13 \\
5 & 0 & 0 & -1 & 1 \\
\hline & $N_{\left(\lambda^{(1)}+\lambda^{(2)}, \lambda^{(1)}\right), g, \beta}^{c=1}$
\end{tabular}

\begin{tabular}{|r|cccc|}
\hline & $\beta=5 / 2$ & $7 / 2$ & $9 / 2$ & $11 / 2$ \\
\hline $\mathrm{g}=0$ & -22 & 136 & -231 & 117 \\
1 & -22 & 231 & -521 & 312 \\
2 & -8 & 173 & -532 & 367 \\
3 & -1 & 67 & -296 & 230 \\
4 & 0 & 13 & -92 & 79 \\
5 & 0 & 1 & -15 & 14 \\
6 & 0 & 0 & -1 & 1 \\
\multicolumn{4}{|c}{$N_{\left(\lambda^{(3)}, \lambda^{(1)}\right), g, \beta}^{c=1}$}
\end{tabular}

$\underline{p_{1}=2, p_{2}=3}$

\begin{tabular}{|l|rr|}
\hline & $\beta=2$ & 3 \\
\hline $\mathrm{g}=0$ & 1 & -1 \\
\hline
\end{tabular}

\begin{tabular}{|r|rrr|}
\hline & $\beta=5 / 2$ & $7 / 2$ & $9 / 2$ \\
\hline $\mathrm{g}=0$ & 1 & -5 & 4 \\
1 & 0 & -1 & 1 \\
\hline
\end{tabular}

$N_{\left(2 \lambda^{(1)}, \lambda^{(1)}\right), g, \beta}^{c=1}$

\begin{tabular}{r|rrr|}
\hline & $\beta=5 / 2$ & $7 / 2$ & $9 / 2$ \\
\hline $\mathrm{g}=0$ & 4 & -13 & 9 \\
1 & 1 & -7 & 6 \\
2 & 0 & -1 & 1 \\
\hline \multicolumn{3}{c}{$N_{\left(\lambda^{(2)}, \lambda^{(1)}\right), g, \beta}^{c=1}$}
\end{tabular}




\begin{tabular}{r|rrrr|}
\hline & $\beta=3$ & 4 & 5 & 6 \\
\hline $\mathrm{g}=0$ & 1 & -15 & 36 & -22 \\
1 & 0 & -7 & 29 & -22 \\
2 & 0 & -1 & 9 & -8 \\
3 & 0 & 0 & 1 & -1 \\
$N_{\left(3 \lambda^{(1)}, \lambda^{(1)}\right), g, \beta}^{c=1}$
\end{tabular}

\begin{tabular}{r|rrrr|}
\hline & $\beta=3$ & 4 & 5 & 6 \\
\hline $\mathrm{g}=0$ & 13 & -106 & 204 & -111 \\
1 & 7 & -118 & 319 & -208 \\
2 & 1 & -55 & 219 & -165 \\
3 & 0 & -12 & 78 & -66 \\
4 & 0 & -1 & 14 & -13 \\
5 & 0 & 0 & 1 & -1 \\
\hline & $N_{\left(\lambda^{(1)}+\lambda^{(2)}, \lambda^{(1)}\right), g, \beta}^{c=1}$
\end{tabular}

\begin{tabular}{r|rrrr|}
\hline & $\beta=3$ & 4 & 5 & 6 \\
\hline $\mathrm{g}=0$ & 22 & -136 & 231 & -117 \\
1 & 22 & -231 & 521 & -312 \\
2 & 8 & -173 & 532 & -367 \\
3 & 1 & -67 & 296 & -230 \\
4 & 0 & -13 & 92 & -79 \\
5 & 0 & -1 & 15 & -14 \\
6 & 0 & 0 & 1 & -1 \\
\hline \multicolumn{4}{|c}{$N_{\left(\lambda^{(3)}, \lambda(1)\right.}^{c=1}, g, \beta$} \\
\end{tabular}

\section{Trefoil \# Hopf Link}

We consider the link whose one component is trefoil and other is Hopf link. We tabulate $N_{R_{1}, R_{2}, g, \beta}^{c=1}$ for the case where both the components carry no framing.

\begin{tabular}{|r|cccc|}
\hline & $\beta=1 / 2$ & $3 / 2$ & $5 / 2$ & $7 / 2$ \\
\hline $\mathrm{g}=0$ & -3 & 6 & -4 & 1 \\
1 & -1 & 2 & -1 & 0 \\
\hline \multicolumn{5}{c}{$N_{\left(\lambda^{(1)}, \lambda^{(1)}\right), g, \beta}^{c=1}$}
\end{tabular}




\begin{tabular}{|r|rrrrrr|}
\hline & $\beta=1$ & 2 & 3 & 4 & 5 & 6 \\
\hline $\mathrm{g}=0$ & -14 & 87 & -218 & 266 & -157 & 36 \\
1 & -11 & 113 & -415 & 635 & -427 & 105 \\
2 & -2 & 55 & -330 & 650 & -485 & 112 \\
3 & 0 & 12 & -132 & 351 & -285 & 54 \\
4 & 0 & 1 & -26 & 104 & -91 & 12 \\
5 & 0 & 0 & -2 & 16 & -15 & 1 \\
6 & 0 & 0 & 0 & 1 & -1 & 0 \\
\hline & $N_{\left(2 \lambda^{(1)}, \lambda(1)\right.}^{c=1}, g, \beta$ & &
\end{tabular}

\begin{tabular}{|r|rrrrrr|}
\hline & $\beta=1$ & 2 & 3 & 4 & 5 & 6 \\
\hline $\mathrm{g}=0$ & -24 & 154 & -363 & 410 & -226 & 49 \\
1 & -26 & 282 & -910 & 1271 & -813 & 196 \\
2 & -9 & 209 & -989 & 1728 & -1233 & 294 \\
3 & -1 & 77 & -572 & 1275 & -989 & 210 \\
4 & 0 & 14 & -182 & 545 & -454 & 77 \\
5 & 0 & 1 & -30 & 135 & -120 & 14 \\
6 & 0 & 0 & -2 & 18 & -17 & 1 \\
7 & 0 & 0 & 0 & 1 & -1 & 0 \\
\hline
\end{tabular}

\section{Summary and Discussions}

In this paper, we have briefly presented framed link invariants in $S O(N)$ Chern-Simons theory. Then, we studied the expectation value of the observables in topological string theory carrying $S O$ holonomy. We had derived modified Frobenius equations leading to new polynomial invariants as a reformulation of framed link invariants in $S O(N)$ Chern-Simons gauge theory. We have proposed new conjectures which are generalisation of the Ooguri-Vafa conjecture and Bouchard-Florea-Marino conjecture involving reformulated $S O(N)$ framed link invariants. We have explicitly computed the reformulated polynomial invariants and BPS integer coefficients, corresponding to cross-cap $c=1$ unoriented topological string amplitudes, for some non-trivial framed knots and framed two-component links verifying the conjecture.

It is still a challenging problem of obtaining cross-cap $c=2$ unoriented string amplitude on an orientifold of a Calabi-Yau background. This requires deriving the amplitude on a covering geometry [17]. 
Another open question is to study $S O(N)$ Chern-Simons free-energy at large $N$ for threemanifolds other than $S^{3}$. In particular, we have to pose new duality conjectures involving topological strings on orientifold background corresponding to $S O(N)$ Chern-Simons theory on orbifolds of $S^{3}$. We hope to study these challenging issues in future. 


\section{Acknowledgments}

PB would like to thank CSIR for the grant. The work of PR is supported by Department of Science and Technology grant under "SERC FAST TRACK Scheme for Young Scientists". 


\section{Appendix}

\section{A Knot and Link Invariants $V_{\Lambda_{R_{1}}, \Lambda_{R_{2}}, \ldots, \Lambda_{R_{r}}}[L](q, \lambda)$}

In this Appendix we present the knot and link invariants for some knots and links with arbitrary framings.

1. Unknot with framing $p$

$$
\begin{aligned}
& V_{\lambda^{(1)}}=(-1)^{p} \lambda^{p / 2}\left[1+\frac{q^{1 / 2} \lambda^{-1 / 2}(-1+\lambda)}{-1+q}\right] \\
& V_{2 \lambda^{(1)}}=\frac{q^{p} \lambda^{p}(-1+\lambda)\left(-q+q^{2} \lambda+q^{1 / 2} \lambda^{1 / 2}\left(-1+q^{2}\right)\right)}{(-1+q)^{2} \lambda(1+q)} \\
& V_{\lambda(2)}=\frac{q^{-p} \lambda^{p}(-1+\lambda)\left(-q^{2}+q \lambda+q^{1 / 2} \lambda^{1 / 2}\left(-1+q^{2}\right)\right)}{(-1+q)^{2} \lambda(1+q)} \\
& V_{3 \lambda^{(1)}}=\frac{(-1)^{p} q^{3 p} \lambda^{3 p / 2}}{(-1+q)^{3}(1+q)\left(1+q+q^{2}\right)}\left[q \lambda ^ { - 3 / 2 } ( - 1 + q \lambda ) \left(q^{1 / 2}+q^{5 / 2} \lambda^{2}\right.\right. \\
& \left.\left.-q^{1 / 2} \lambda\left(1+q^{2}\right)-\lambda^{1 / 2}\left(-1+q^{3}\right)+\lambda^{3 / 2}\left(-1+q^{3}\right)\right)\right] \\
& V_{\lambda^{(1)}+\lambda^{(2)}}=\frac{(-1)^{p} \lambda^{3 p / 2}(-q+\lambda)(-1+q \lambda)\left(-q^{3 / 2}+q^{3 / 2} \lambda+\lambda^{1 / 2}\left(-1+q^{3}\right)\right)}{(-1+q)^{3} \lambda^{3 / 2}\left(1+q+q^{2}\right)} \\
& V_{\lambda^{(3)}}=\frac{(-1)^{p} q^{-3 p} \lambda^{3 p / 2}}{(-1+q)^{3}(1+q)\left(1+q+q^{2}\right)}\left[q \lambda ^ { - 3 / 2 } ( - q + \lambda ) \left(q^{5 / 2}+q^{1 / 2} \lambda^{2}\right.\right. \\
& \left.\left.-q^{1 / 2} \lambda\left(1+q^{2}\right)-\lambda^{1 / 2}\left(-1+q^{3}\right)+\lambda^{3 / 2}\left(-1+q^{3}\right)\right)\right] \\
& V_{4 \lambda^{(1)}}=\frac{q^{6 p} \lambda^{2 p}}{(-1+q)^{4} \lambda^{2}(1+q)^{2}\left(1+q^{2}\right)\left(1+q+q^{2}\right)}[(-1+\lambda)(-1+q \lambda) \\
& \left.\left(-1+q^{2} \lambda\right)\left(-q^{2}+q^{5} \lambda+q^{3 / 2} \lambda^{1 / 2}\left(-1+q^{4}\right)\right)\right] \\
& V_{2 \lambda^{(1)}+\lambda^{(2)}}=\frac{q^{2 p} \lambda^{2 p}}{(-1+q)^{4}(1+q)^{2}\left(1+q^{2}\right)}\left[q^{1 / 2} \lambda^{-2}\left(-q^{1 / 2}+\lambda^{1 / 2}\right)\left(q^{1 / 2}+\lambda^{1 / 2}\right)\right. \\
& \left.\left(q^{3 / 2}+\lambda^{1 / 2}\right)(-1+\lambda)\left(-1+q^{2} \lambda\right)\left(-1+q^{5 / 2} \lambda^{1 / 2}\right)\right]
\end{aligned}
$$




$$
\begin{aligned}
V_{2 \lambda^{(2)}}= & \frac{\lambda^{2 p}}{(-1+q)^{4} \lambda^{2}(1+q)^{2}\left(1+q+q^{2}\right)}\left[q^{4}+q^{4} \lambda^{4}-q^{3} \lambda^{3}(1+q)^{2}\right. \\
& -q^{3} \lambda(1+q)^{2}-(-1+q) q^{5 / 2} \lambda^{1 / 2}(1+q)^{2} \\
& +(-1+q) q^{5 / 2} \lambda^{7 / 2}(1+q)^{2} \\
& +(-1+q) q^{1 / 2} \lambda^{3 / 2}(1+q)^{2}(1+(-1+q) q)\left(1+q+q^{2}\right) \\
& -(-1+q) q^{1 / 2} \lambda^{5 / 2}(1+q)^{2}(1+(-1+q) q)\left(1+q+q^{2}\right) \\
& -\lambda^{2}\left(1+q+q^{2}\right)(1+q(-2+q(-1+(-2+q)(-1+q) \\
& q(1+q))))] \\
V_{\lambda^{(1)}+\lambda^{(3)}=} & \frac{q^{-2 p} \lambda^{2 p}}{(-1+q)^{4}(1+q)^{2}\left(1+q^{2}\right)}\left[q^{1 / 2} \lambda^{-2}\left(q^{5 / 2}+\lambda^{1 / 2}\right)(-1+\lambda)\right. \\
& \left.\left(-q^{2}+\lambda\right)\left(-1+q^{1 / 2} \lambda^{1 / 2}\right)\left(1+q^{1 / 2} \lambda^{1 / 2}\right)\left(-1+q^{3 / 2} \lambda^{1 / 2}\right)\right] \\
V_{\lambda^{(4)}}= & \frac{q^{-6 p} \lambda^{2 p}}{(-1+q)^{4} \lambda^{2}(1+q)^{2}\left(1+q^{2}\right)\left(1+q+q^{2}\right)}[(-1+\lambda)(-q+\lambda) \\
& \left.\left(-q^{2}+\lambda\right)\left(-q^{5}+q^{2} \lambda+q^{3 / 2} \lambda^{1 / 2}\left(-1+q^{4}\right)\right)\right]
\end{aligned}
$$

\section{Trefoil knot with framing $p$}

$$
\begin{aligned}
V_{\lambda^{(1)}}= & \frac{(-1)^{p}}{(-1+q)^{2}(1+q)}\left[q ^ { - 2 } \lambda ^ { ( p + 1 ) / 2 } \left(q^{3 / 2}-q^{11 / 2}+q^{6} \lambda^{1 / 2}-q^{3 / 2} \lambda\right.\right. \\
& +q \lambda^{1 / 2}+q^{3} \lambda^{3 / 2}+q^{5} \lambda^{5 / 2}-q^{5 / 2} \lambda-q^{6} \lambda^{3 / 2}+q^{9 / 2} \lambda+q^{11 / 2} \lambda \\
& -q^{3} \lambda^{1 / 2}-q^{4} \lambda^{1 / 2}-q \lambda^{3 / 2}+q^{4} \lambda^{3 / 2}+q^{5 / 2} \lambda^{2}-q^{9 / 2} \lambda^{2}+q^{2} \lambda^{5 / 2} \\
& \left.\left.-q^{3} \lambda^{5 / 2}-q^{4} \lambda^{5 / 2}\right)\right] \\
V_{2 \lambda^{(1)}}= & \frac{1}{(-1+q)^{2}(1+q)}\left[q ^ { p - 3 } \lambda ^ { p + 1 } \left(q^{2}+q^{5}+q^{6}+q^{8}+(-1+q)^{2} q^{9} \lambda^{5}\right.\right. \\
& (1+q)-q^{19 / 2} \lambda^{9 / 2}\left(-1+q^{2}\right)+q^{10} \lambda^{4}\left(1+q-q^{3}\right)-q^{2} \lambda(1+q) \\
& \left(1+q^{2}\right)\left(1+q^{3}+q^{4}\right)+(-1+q) q^{3 / 2} \lambda^{3 / 2}(1+q)(1+(-1+q) q) \\
& \left(1+q+q^{2}\right)\left(1+q+q^{2}+q^{3}+q^{4}\right)-(-1+q) q^{5 / 2} \lambda^{5 / 2}(1+q) \\
& \left(1+q^{2}\right)\left(1+q+q^{2}+q^{3}+q^{4}+q^{5}+q^{6}\right)+q^{5} \lambda^{3}(1+q) \\
& \left(-1-q^{2}-q^{4}-q^{6}+q^{7}\right)-q^{3 / 2} \lambda^{1 / 2}\left(-1-q^{3}+q^{7}+q^{8}\right) \\
& +q^{9 / 2} \lambda^{7 / 2}\left(-1-q^{3}+q^{7}+q^{8}\right)-q^{3} \lambda^{2}(1+(-1+q) q)
\end{aligned}
$$




$$
\begin{aligned}
& \left.\left.\left(-1+q\left(-2+q\left(1+q^{2}\right)\left(-3-3 q+q^{3}\right)\right)\right)\right)\right] \\
V_{\lambda^{(2)}}= & \frac{1}{(-1+q)^{2}(1+q)}\left[q ^ { - ( 2 1 / 2 + p ) } \lambda ^ { p + 1 } \left((-1+q)^{2} q^{9 / 2} \lambda^{5}(1+q)\right.\right. \\
& -q^{5} \lambda^{9 / 2}\left(-1+q^{2}\right)+q^{7 / 2} \lambda^{4}\left(-1+q^{2}+q^{3}\right)-q^{15 / 2} \lambda(1+q) \\
& \left(1+q^{2}\right)\left(1+q+q^{4}\right)+(-1+q) q^{5} \lambda^{3 / 2}(1+q)(1+(-1+q) q) \\
& \left(1+q+q^{2}\right)\left(1+q+q^{2}+q^{3}+q^{4}\right)+q^{17 / 2}\left(1+q^{2}+q^{3}+q^{6}\right) \\
& -(-1+q) q^{4} \lambda^{5 / 2}(1+q)\left(1+q^{2}\right)\left(1+q+q^{2}+q^{3}+q^{4}+q^{5}+q^{6}\right) \\
& -q^{7 / 2} \lambda^{3}(1+q)\left(-1+q+q^{3}+q^{5}+q^{7}\right)-q^{7} \lambda^{1 / 2}\left(-1-q+q^{5}+q^{8}\right) \\
& +q^{4} \lambda^{7 / 2}\left(-1-q+q^{5}+q^{8}\right)+q^{9 / 2} \lambda^{2}(-1+q(1+q(1+q \\
& \left.\left.\left.\left.\left(1+q+q^{2}\right)\left(1+q+q^{2}+q^{4}\right)\right)\right)\right)\right]
\end{aligned}
$$

\section{Hopf Link with framing $p_{1}$ on first strand and $p_{2}$ on the second}

$$
\begin{aligned}
V_{\lambda^{(1)} \lambda^{(1)}=} & \frac{1}{(-1+q)^{2}}\left[(-1)^{p_{1}+p_{2}} q^{-1 / 2} \lambda^{\left(p_{1}+p_{2}-2\right) / 2}\left(q^{1 / 2}+\lambda^{1 / 2}\right)\right. \\
& \left(-1+q^{1 / 2} \lambda^{1 / 2}\right)\left(-1+q-q^{2}+(-1+q) q^{1 / 2} \lambda^{1 / 2}\right. \\
& +\lambda(1+(-1+q) q)] \\
V_{2 \lambda^{(1)} \lambda^{(1)}=} & \frac{1}{(-1+q)^{3}(1+q)}\left[(-1)^{p_{2}} q^{-1+p_{1}} \lambda^{\left(2 p_{1}+p_{2}-3\right) / 2}\left(-1+\lambda^{1 / 2}\right)\right. \\
& \left(1+\lambda^{1 / 2}\right)\left(q^{1 / 2}+\lambda^{1 / 2}\right)\left(-1+q^{1 / 2} \lambda^{1 / 2}\right)\left(-1+q^{3 / 2} \lambda^{1 / 2}\right) \\
& \left.\left(1-q+q^{3}+q^{1 / 2} \lambda^{1 / 2}\left(1+(-1+q) q^{2}\right)\right)\right] \\
V_{\lambda^{(2)} \lambda^{(1)}=} & (-1+q)^{3}(1+q)\left[(-1)^{p_{2}} q^{-\left(2+p_{1}\right)} \lambda^{\left(2 p_{1}+p_{2}-3\right) / 2}\right. \\
& \left(-\left(q^{7 / 2}\left(1+(-1+q) q^{2}\right)\right)+q^{3 / 2} \lambda^{3}\left(1-q+q^{3}\right)\right. \\
& +q^{5 / 2} \lambda\left(1+q+q^{4}\right)+(-1+q) q^{2} \lambda^{1 / 2}\left(1+q+q^{4}\right) \\
& -q^{3 / 2} \lambda^{2}\left(1+q^{3}+q^{4}\right)+(-1+q) q \lambda^{5 / 2}\left(1+q^{3}+q^{4}\right) \\
& \left.\left.-q \lambda^{3 / 2}\left(-1+q^{6}\right)\right)\right] \\
V_{3 \lambda^{(1)} \lambda^{(1)}=} & \frac{1}{(-1+q)^{4}(1+q)\left(1+q+q^{2}\right)}\left[(-1)^{3 p_{1}+p_{2}} q^{-3+3 p_{1}} \lambda^{\left(3 p_{1}+p_{2}-4\right) / 2}\right. \\
&
\end{aligned}
$$




$$
\begin{aligned}
& (-1+\lambda)\left(q^{7} \lambda^{3}\left(1+(-1+q) q^{3}\right)-q^{2}\left(1-q+q^{4}\right)\right. \\
& -q^{5} \lambda^{2}\left(1+q+q^{5}\right)+q^{3} \lambda\left(1+q^{4}+q^{5}\right)+(-1+q) q^{9 / 2} \lambda^{5 / 2} \\
& \left(1+q+q^{2}+q^{6}\right)+q^{3 / 2} \lambda^{1 / 2}\left(-1+q-q^{4}+q^{7}\right) \\
& \left.\left.-q^{5 / 2} \lambda^{3 / 2}\left(-1+q^{8}\right)\right)\right] \\
& V_{\lambda^{(1)}+\lambda^{(2)} \lambda^{(1)}}=\frac{1}{(-1+q)^{4}(1+q)^{2}\left(1+q+q^{2}\right)}\left[(-1)^{3 p_{1}+p_{2}} q^{-2} \lambda^{\left(3 p_{1}+p_{2}-4\right) / 2}\right. \\
& \left(q^{3}+q^{4}+q^{8}+q^{9}-q^{2} \lambda(1+q)^{2}\left(1+q^{2}\right)\left(1+q^{4}\right)\right. \\
& -q^{2} \lambda^{3}(1+q)^{2}\left(1+q^{2}\right)\left(1+q^{4}\right)+(-1+q) q^{3 / 2} \lambda^{7 / 2}(1+q)^{2} \\
& \left(1+q^{2}\right)\left(1+q^{4}\right)+q^{2} \lambda^{2}(1+q)^{2}\left(1+q+q^{2}\right)\left(1+q^{4}\right) \\
& +(-1+q) q^{1 / 2} \lambda^{3 / 2}(1+q)^{2}\left(1+q^{2}\right)\left(1+q+q^{2}\right)\left(1+q^{4}\right) \\
& -(-1+q) q^{1 / 2} \lambda^{5 / 2}(1+q)^{2}\left(1+q^{2}\right)\left(1+q+q^{2}\right)\left(1+q^{4}\right) \\
& \left.\left.+q^{3} \lambda^{4}\left(1+q+q^{5}+q^{6}\right)-q^{3 / 2} \lambda^{1 / 2}\left(-1-q+q^{8}+q^{9}\right)\right)\right] \\
& V_{\lambda^{(3)} \lambda^{(1)}}=\frac{1}{(-1+q)^{4}(1+q)\left(1+q+q^{2}\right)}\left[(-1)^{3 p_{1}+p_{2}} q^{-2-3 p_{1}} \lambda^{\left(-4+3 p_{1}+p_{2}\right) / 2}\right. \\
& (-1+\lambda)\left(-\left(q^{6}\left(1+(-1+q) q^{3}\right)\right)+q \lambda^{3}\left(1-q+q^{4}\right)\right. \\
& +q^{4} \lambda\left(1+q+q^{5}\right)-q^{2} \lambda^{2}\left(1+q^{4}+q^{5}\right)+(-1+q) q^{7 / 2} \lambda^{1 / 2} \\
& \left(1+q+q^{2}+q^{6}\right)+q^{1 / 2} \lambda^{5 / 2}\left(-1+q-q^{4}+q^{7}\right) \\
& \left.\left.-q^{3 / 2} \lambda^{3 / 2}\left(-1+q^{8}\right)\right)\right] \\
& V_{2 \lambda^{(1)} 2 \lambda^{(1)}}=\frac{1}{(-1+q)^{4}(1+q)^{2}}\left[q^{-4+p_{1}+p_{2}} \lambda^{-2+p_{1}+p_{2}}\right. \\
& \left(-\left(q^{2} \lambda(1+q)\left(1-q+q^{3}+q^{6}\right)\right)-(-1+q) q^{3 / 2} \lambda^{1 / 2}(1+q)\right. \\
& \left(1-q+q^{3}+q^{6}\right)-q^{5} \lambda^{3}(1+q)\left(1+q^{3}-q^{5}+q^{6}\right)+(-1+q) \\
& q^{9 / 2} \lambda^{7 / 2}(1+q)\left(1+q^{3}-q^{5}+q^{6}\right)-(-1+q) q^{5 / 2} \lambda^{5 / 2}(1+q) \\
& \left(1+q^{2}+q^{3}+q^{4}+q^{5}+q^{8}\right)+q^{3} \lambda^{2}\left(1+q^{3}+2 q^{4}+q^{5}+q^{8}\right) \\
& +(-1+q) q^{3 / 2} \lambda^{3 / 2}(1+q)\left(1+q^{3}+q^{4}+q^{5}+q^{6}+q^{8}\right) \\
& +q^{6} \lambda^{4}\left(1+(-1+q) q(1+q)\left(1+(-1+q) q^{2}\right)\right) \\
& \left.\left.+q^{2}\left(1+(-1+q) q(1+q)\left(1+q-q^{2}+q^{3}\right)\right)\right)\right]
\end{aligned}
$$




$$
\begin{aligned}
& V_{2 \lambda^{(1)} \lambda^{(2)}}=\frac{1}{(-1+q)^{4}(1+q)^{2}}\left[q ^ { - 2 + p _ { 1 } - p _ { 2 } } \lambda ^ { - 2 + p _ { 1 } + p _ { 2 } } ( - 1 + \lambda ) \left(-q^{3}+q^{5}-q^{7}\right.\right. \\
& -(-1+q) q^{1 / 2} \lambda^{3 / 2}(1+q)^{2}(1+(-1+q) q)\left(1+q^{4}\right)+q^{3} \lambda^{3} \\
& \left(1-q^{2}+q^{4}\right)-q^{2} \lambda^{2}\left(1+q^{3}+q^{6}\right)+q^{2} \lambda\left(1+q^{3}+q^{6}\right) \\
& +q^{3 / 2} \lambda^{1 / 2}\left(-1+q^{2}-q^{5}+q^{7}\right)+(-1+q) q^{3 / 2} \lambda^{5 / 2}(1+q)^{2} \\
& \left.\left.\left(1+(-1+q) q\left(1+q^{2}\right)\right)\right)\right] \\
& V_{\lambda^{(2)} \lambda^{(2)}}=\frac{1}{(-1+q)^{4}(1+q)^{2}}\left[q ^ { - 9 / 2 - p _ { 1 } - p _ { 2 } } \lambda ^ { - 2 + p _ { 1 } + p _ { 2 } } \left(-\left(q^{5 / 2} \lambda^{3}(1+q)\right.\right.\right. \\
& \left.\left(1-q+q^{3}+q^{6}\right)\right)+(-1+q) q^{2} \lambda^{7 / 2}(1+q)\left(1-q+q^{3}+q^{6}\right) \\
& -(-1+q) q^{5} \lambda^{1 / 2}(1+q)\left(1+q^{3}-q^{5}+q^{6}\right)-q^{11 / 2} \lambda(1+q) \\
& \left(1+q^{3}-q^{5}+q^{6}\right)+\left(-1+q^{2}\right) q^{3} \lambda^{3 / 2}\left(1+q^{2}+q^{3}+q^{4}+q^{5}+q^{8}\right) \\
& +q^{7 / 2} \lambda^{2}\left(1+q^{3}+2 q^{4}+q^{5}+q^{8}\right)-(-1+q) q^{2} \lambda^{5 / 2}(1+q) \\
& \left(1+q^{3}+q^{4}+q^{5}+q^{6}+q^{8}\right)+q^{13 / 2}(1+(-1+q) q(1+q) \\
& \left.\left(1+(-1+q) q^{2}\right)\right)+q^{5 / 2} \lambda^{4}(1+(-1+q) q(1+q) \\
& \left.\left.\left.\left(1+q-q^{2}+q^{3}\right)\right)\right)\right]
\end{aligned}
$$

\section{B $S O(N)$ Reformulated Invariants $g_{R_{1}, R_{2}, \ldots, R_{r}}(q, \lambda)$}

\section{Trefoil with framing $p$}

$$
\begin{aligned}
g_{\lambda^{(1)}}(q, \lambda)= & \frac{1}{(-1+q) q}\left[( - 1 ) ^ { p } \lambda ^ { ( 1 + p ) / 2 } \left(-\left(q^{1 / 2}\left(1+q^{2}\right)\right)+\left(-1+q^{3}\right) \lambda^{1 / 2}\right.\right. \\
& +q^{1 / 2}\left(1+q+q^{2}\right) \lambda-\left(-1+q^{3}\right) \lambda^{3 / 2}-q^{3 / 2} \lambda^{2} \\
& \left.\left.+(-1+q) q \lambda^{5 / 2}\right)\right] \\
g_{2 \lambda^{(1)}}(q, \lambda)= & \frac{1}{2(-1+q)^{2} q^{2}(1+q)}\left[2(-1+q)^{2} q^{2}(1+q)+\lambda^{1+p} \times\right. \\
& \left(-2 q^{p}\left(-\left(q+q^{4}+q^{5}+q^{7}\right)+q^{1 / 2}\left(-1-q^{3}+q^{7}+q^{8}\right) \lambda^{1 / 2}\right.\right. \\
& +q(1+q)\left(1+q^{2}\right)\left(1+q^{3}+q^{4}\right) \lambda-(-1+q) q^{1 / 2}(1+q) \\
& (1+(-1+q) q)\left(1+q+q^{2}\right)\left(1+q+q^{2}+q^{3}+q^{4}\right) \lambda^{3 / 2} \\
& +q^{2}(1+(-1+q) q)\left(-1+q\left(-2+q\left(1+q^{2}\right)\right.\right.
\end{aligned}
$$




$$
\begin{aligned}
& \left.\left.\left(-3-3 q+q^{3}\right)\right)\right) \lambda^{2}+(-1+q) q^{3 / 2}(1+q)\left(1+q^{2}\right) \\
& \left(1+q+q^{2}+q^{3}+q^{4}+q^{5}+q^{6}\right) \lambda^{5 / 2}-q^{4}(1+q) \\
& \left(-1-q^{2}-q^{4}-q^{6}+q^{7}\right) \lambda^{3}-q^{7 / 2}\left(-1-q^{3}+q^{7}+q^{8}\right) \lambda^{7 / 2} \\
& +q^{9}\left(-1-q+q^{3}\right) \lambda^{4}+q^{17 / 2}\left(-1+q^{2}\right) \lambda^{9 / 2} \\
& \left.-(-1+q)^{2} q^{8}(1+q) \lambda^{5}\right)+(-1)^{p}\left(-\left((-1)^{p} \times\right.\right. \\
& (1+q)\left(q^{1 / 2}\left(1+q^{2}\right)-\left(-1+q^{3}\right) \lambda^{1 / 2}-q^{1 / 2}\left(1+q+q^{2}\right) \lambda\right. \\
& \left.\left.+\left(-1+q^{3}\right) \lambda^{3 / 2}+q^{3 / 2} \lambda^{2}-(-1+q) q \lambda^{5 / 2}\right)^{2}\right)+(-1+q)(q+\lambda) \\
& \left.\left.\left.(-1+q \lambda)\left(-1+(q-\lambda)\left(-\lambda-q \lambda^{2}+q^{3}\left(-1+\lambda^{2}\right)\right)\right)\right)\right)\right] \\
& g_{\lambda^{(2)}}(q, \lambda)=\frac{1}{2(-1+q)^{2}(1+q)}\left[q ^ { - 7 - p } \lambda ^ { 1 + p } \left(2 \left(q^{5}+q^{7}+q^{8}+q^{11}\right.\right.\right. \\
& -q^{7 / 2}\left(-1-q+q^{5}+q^{8}\right) \lambda^{1 / 2}-q^{4}(1+q)\left(1+q^{2}\right)\left(1+q+q^{4}\right) \lambda \\
& +(-1+q) q^{3 / 2}(1+q)(1+(-1+q) q)\left(1+q+q^{2}\right) \\
& \left(1+q+q^{2}+q^{3}+q^{4}\right) \lambda^{3 / 2}+q\left(-1+q\left(1+q\left(1+q\left(1+q+q^{2}\right)\right.\right.\right. \\
& \left.\left.\left.\left(1+q+q^{2}+q^{4}\right)\right)\right)\right) \lambda^{2}-(-1+q) q^{1 / 2}(1+q)\left(1+q^{2}\right) \\
& \left(1+q+q^{2}+q^{3}+q^{4}+q^{5}+q^{6}\right) \lambda^{5 / 2}-\left(-1+q+q^{3}+q^{5}+q^{7}\right) \lambda^{3} \\
& (1+q)+q^{1 / 2}\left(-1-q+q^{5}+q^{8}\right) \lambda^{7 / 2}+\left(-1+q^{2}+q^{3}\right) \lambda^{4} \\
& \left.-q^{3 / 2}\left(-1+q^{2}\right) \lambda^{9 / 2}+(-1+q)^{2} q(1+q) \lambda^{5}\right) \\
& -(-1)^{p} q^{5+p}\left(( - 1 ) ^ { p } ( 1 + q ) \left(q^{1 / 2}\left(1+q^{2}\right)-\left(-1+q^{3}\right) \lambda^{1 / 2}\right.\right. \\
& -q^{1 / 2}\left(1+q+q^{2}\right) \lambda+\left(-1+q^{3}\right) \lambda^{3 / 2}+q^{3 / 2} \lambda^{2} \\
& \left.-(-1+q) q \lambda^{5 / 2}\right)^{2}+(-1+q)(q+\lambda)(-1+q \lambda)(-1+(q-\lambda) \\
& \left.\left.\left.\left.\left(-\lambda-q \lambda^{2}+q^{3}\left(-1+\lambda^{2}\right)\right)\right)\right)\right)\right]
\end{aligned}
$$

\section{Connected sum of trefoil and trefoil with framing $p$}

$$
\begin{aligned}
g_{\lambda^{(1)}}(q, \lambda)= & \frac{1}{(-1+q) q^{2}}\left[(-1)^{p} \lambda^{(3+p) / 2}\left(q^{1 / 2}+\lambda^{1 / 2}\right)\left(-1+q^{1 / 2} \lambda^{1 / 2}\right)\right. \\
& \left.\left(-1+\left(q^{1 / 2}-\lambda^{1 / 2}\right)\left(q^{1 / 2}(q(-1+\lambda)-\lambda)-\lambda^{1 / 2}\right)\right)^{2}\right] \\
g_{2 \lambda^{(1)}}(q, \lambda)= & \frac{1}{2(-1+q)^{2} q^{4}(1+q)}\left[2(-1+q)^{2} q^{4}(1+q)\right.
\end{aligned}
$$




$$
\begin{aligned}
& +\lambda^{3+p}\left(-\left(\left(-1+\left(q^{1 / 2}-\lambda^{1 / 2}\right)\left(q^{1 / 2}(q(-1+\lambda)-\lambda)-\lambda^{1 / 2}\right)\right)^{4}\right.\right. \\
& \left.(1+q)\left(q^{1 / 2}+\lambda^{1 / 2}\right)^{2}\left(-1+q^{1 / 2} \lambda^{1 / 2}\right)^{2}\right)+2 q^{1 / 2+p}\left(q^{1 / 2}+\lambda^{1 / 2}\right) \\
& \left(-1+q^{3 / 2} \lambda^{1 / 2}\right)(-1+\lambda)\left[\left(1+q^{3}\left(1+q+q^{3}\right)\right.\right. \\
& -(-1+q) q^{3 / 2}(1+q)^{2}(1+(-1+q) q) \lambda^{1 / 2} \\
& -q(1+q)^{2}\left(1-q+2 q^{3}-2 q^{4}+q^{5}\right) \lambda+(-1+q) q^{5 / 2}(1+q) \\
& \left(1+q-q^{2}+q^{3}+q^{4}\right) \lambda^{3 / 2}+q^{3}(1+(-1+q) q(1+q) \\
& \left.\left(1+(-1+q)^{2} q\right)\right) \lambda^{2}-q^{9 / 2}\left(-1+q+q^{2}-2 q^{3}+q^{5}\right) \lambda^{5 / 2} \\
& \left.\left.+(-1+q)^{2} q^{6}(1+q) \lambda^{3}\right)\right]^{2}-(-1)^{p}(-1+q)(q+\lambda) \\
& \left.\left.(-1+q \lambda)\left(-1+(q-\lambda)\left(-\lambda-q \lambda^{2}+q^{3}\left(-1+\lambda^{2}\right)\right)\right)^{2}\right)\right]
\end{aligned}
$$

$$
\begin{aligned}
g_{\lambda(2)}(q, \lambda)= & \frac{1}{2(-1+q)^{2}(1+q)}\left[q ^ { - 5 7 / 2 - p } \lambda ^ { 3 + p } \left(2 q^{14}\left(-1+\lambda^{1 / 2}\right)\left(1+\lambda^{1 / 2}\right)\right.\right. \\
& \left(q^{3 / 2}+\lambda^{1 / 2}\right)\left(-1+q^{1 / 2} \lambda^{1 / 2}\right)\left[\left(q^{7 / 2}\left(1+q^{2}+q^{3}+q^{6}\right)-(-1+q)\right.\right. \\
& q^{3}(1+q)^{2}(1+(-1+q) q) \lambda^{1 / 2}-q^{3 / 2}(1+q)^{2}(1+(-1+q) q) \\
& \left(1-q+q^{3}\right) \lambda+(-1+q) q(1+q)\left(1+q-q^{2}+q^{3}+q^{4}\right) \lambda^{3 / 2} \\
& +q^{1 / 2}\left(1+(-1+q) q(1+q)^{2}(2+(-2+q) q)\right) \lambda^{2} \\
& \left.\left.+\left(1+q^{2}\left(-2+q+q^{2}-q^{3}\right)\right) \lambda^{5 / 2}+(-1+q)^{2} q^{1 / 2}(1+q) \lambda^{3}\right)\right]^{2} \\
& +q^{49 / 2+p}\left(-\left(\left(-1+\left(q^{1 / 2}-\lambda^{1 / 2}\right)\left(q^{1 / 2}(q(-1+\lambda)-\lambda)-\lambda^{1 / 2}\right)\right)^{4}\right.\right. \\
& \left.(1+q)\left(q^{1 / 2}+\lambda^{1 / 2}\right)^{2}\left(-1+q^{1 / 2} \lambda^{1 / 2}\right)^{2}\right)+(-1)^{p}(-1+q)(q+\lambda) \\
& \left.\left.\left.(-1+q \lambda)\left(-1+(q-\lambda)\left(-\lambda-q \lambda^{2}+q^{3}\left(-1+\lambda^{2}\right)\right)\right)^{2}\right)\right)\right]
\end{aligned}
$$

\section{Hopf Link with framing $p_{1}$ on first strand and $p_{2}$ on the second}

$$
\begin{aligned}
g_{\lambda^{(1)}, \lambda^{(1)}}(q, \lambda)= & (-1)^{p_{1}+p_{2}} q^{-1 / 2}\left(q^{1 / 2}+\lambda^{1 / 2}\right)\left(-1+q^{1 / 2} \lambda^{1 / 2}\right) \\
& (-1+\lambda) \lambda^{\left(-2+p_{1}+p_{2}\right) / 2}
\end{aligned}
$$




$$
\begin{aligned}
& \left.+\left(1-q+q^{3}+q^{1 / 2}\left(1+(-1+q) q^{2}\right) \lambda^{1 / 2}\right)\right) \\
& \left.\left(-1+q^{3 / 2} \lambda^{1 / 2}\right)\right)\left(q^{1 / 2}+\lambda^{1 / 2}\right)\left(-1+q^{1 / 2} \lambda^{1 / 2}\right) \\
& \left.(-1+\lambda) \lambda^{\left(-3+2 p_{1}+p_{2}\right) / 2}\right] \\
& g_{\lambda^{(2)}, \lambda^{(1)}}(q, \lambda)=\frac{-1}{(-1+q)^{3}(1+q)}\left[( - 1 ) ^ { p _ { 2 } } q ^ { - 1 - p _ { 1 } } \left((-1+q)^{2} q^{1 / 2+p_{1}}(1+q)\right.\right. \\
& \left(q^{1 / 2}+\lambda^{1 / 2}\right)\left(-1+q^{1 / 2} \lambda^{1 / 2}\right)+\left(q^{3 / 2}+\lambda^{1 / 2}\right)\left(-q^{3 / 2}+\right. \\
& \left.\left.\left(q^{1 / 2}\left(1+(-1+q) q^{2}\right)+\left(-1+q-q^{3}\right) \lambda^{1 / 2}\right)+q^{2} \lambda^{1 / 2}\right)\right) \\
& \left.\left(q^{1 / 2}+\lambda^{1 / 2}\right)\left(-1+q^{1 / 2} \lambda^{1 / 2}\right)(-1+\lambda) \lambda^{\left(-3+2 p_{1}+p_{2}\right) / 2}\right] \\
& g_{3 \lambda^{(1)}, \lambda^{(1)}}(q, \lambda)=\frac{1}{(-1+q)^{2} q^{5 / 2}(1+q)}\left[(-1)^{p_{1}+p_{2}}\left(q^{1 / 2}+\lambda^{1 / 2}\right)\left(-1+q^{1 / 2} \lambda^{1 / 2}\right)\right. \\
& (-1+\lambda) \lambda^{\left(-4+3 p_{1}+p_{2}\right) / 2}\left(q^{2}(1+q)\left(q^{1 / 2}+\lambda^{1 / 2}\right)^{2}\left(-1+q^{1 / 2} \lambda^{1 / 2}\right)^{2}\right. \\
& +q^{1+3 p_{1}}\left(1+q^{1 / 2} \lambda^{1 / 2}\right)\left(-1+q \lambda^{1 / 2}\right)\left(1+q \lambda^{1 / 2}\right)\left(-1+q^{5 / 2} \lambda^{1 / 2}\right) \\
& \left.\left.-q^{3 / 2+p_{1}}\left(q^{1 / 2}+\lambda^{1 / 2}\right)\left(-1+q^{3 / 2} \lambda^{1 / 2}\right)(-1-2 q+q(2+q) \lambda)\right)\right]
\end{aligned}
$$

$$
\begin{aligned}
g_{\lambda^{(1)}+\lambda^{(2)}, \lambda^{(1)}}(q, \lambda)= & \frac{-1}{(-1+q)^{2}(1+q)}\left[(-1)^{p_{1}+p_{2}} q^{-3 / 2-p_{1}}\left(q^{1 / 2}+\lambda^{1 / 2}\right)\right. \\
& \left(-1+q^{1 / 2} \lambda^{1 / 2}\right)(-1+\lambda) \lambda^{\left(-4+3 p_{1}+p_{2}\right) / 2}\left(-\left(q^{1 / 2}\left(q^{3 / 2}+\lambda^{1 / 2}\right)\right.\right. \\
& \left.\left(-1+q^{1 / 2} \lambda^{1 / 2}\right)(q(2+q-2 \lambda)-\lambda)\right)+q^{1 / 2+2 p_{1}}\left(q^{1 / 2}+\lambda^{1 / 2}\right) \\
& \left(-1+q^{3 / 2} \lambda^{1 / 2}\right)(-1-2 q+q(2+q) \lambda)+q^{p_{1}}(1+q) \\
& \left(-3 q^{2}+(-1+q) q^{1 / 2}(1+q(4+q)) \lambda^{1 / 2}\right. \\
& +(1+q(-3+q(10+(-3+q) q))) \lambda \\
& \left.\left.\left.-(-1+q) q^{1 / 2}(1+q(4+q)) \lambda^{3 / 2}-3 q^{2} \lambda^{2}\right)\right)\right]
\end{aligned}
$$

$$
\begin{aligned}
g_{\lambda^{(3)}, \lambda^{(1)}}(q, \lambda)= & \frac{1}{(-1+q)^{2}(1+q)}\left[(-1)^{p 1+p 2} q^{-3 / 2-3 p 1}\left(-1+q^{1 / 2} \lambda^{1 / 2}\right)(-1+\lambda)\right. \\
& \lambda^{(-4+3 p 1+p 2) / 2}\left(q^{1+3 p 1}(1+q)\left(q^{1 / 2}+\lambda^{1 / 2}\right)^{3}\left(-1+q^{1 / 2} \lambda^{1 / 2}\right)^{2}\right. \\
& +q^{1 / 2+2 p 1}\left(q^{1 / 2}+\lambda^{1 / 2}\right)\left(q^{3 / 2}+\lambda^{1 / 2}\right)\left(-1+q^{1 / 2} \lambda^{1 / 2}\right) \\
& (q(2+q-2 \lambda)-\lambda)+\left(q^{2}-\lambda\right)\left(q^{7 / 2}+q \lambda^{1 / 2}-q^{5 / 2} \lambda-\lambda^{3 / 2}\right)(B .12)
\end{aligned}
$$




$$
\begin{aligned}
g_{2 \lambda^{(1)}, 2 \lambda^{(1)}}(q, \lambda)= & \frac{1}{2(-1+q)^{4} q(1+q)}\left[\lambda ^ { - 2 + p 1 + p 2 } \left(\left(q^{1 / 2}+\lambda^{1 / 2}\right)^{2}\left(-1+q^{1 / 2} \lambda^{1 / 2}\right)^{2}\right.\right. \\
& \left(2 q^{3 / 2+p 2}\left(q^{1 / 2}+\lambda^{1 / 2}\right)\left(-1+q^{3 / 2} \lambda^{1 / 2}\right)(-1+\lambda)+2 q^{p 1}(-1+\lambda)\right. \\
& \left(-q^{2}+q^{3 / 2}\left(-1+q^{2}\right) \lambda^{1 / 2}+q^{3} \lambda\right)-(1+q)(-1-(-5+q) q \\
& \left.-3(-1+q) q^{1 / 2} \lambda^{1 / 2}+(1+(-5+q) q) \lambda\right)\left(-1+q-q^{2}\right. \\
& \left.\left.+(-1+q) q^{1 / 2} \lambda^{1 / 2}+(1+(-1+q) q) \lambda\right)\right)+(-1)^{p 1+p 2}(q+1) \\
& \left(-3(-1)^{p 1+p 2} q\left(q^{1 / 2}+\lambda^{1 / 2}\right)^{4}\left(-1+q^{1 / 2} \lambda^{1 / 2}\right)^{4}-(-1+q)^{4}(q+\lambda)\right. \\
& \left.(-1+q \lambda)\left(-1+\lambda^{2}\right)\right)+2\left(-1+\lambda^{1 / 2}\right)\left(1+\lambda^{1 / 2}\right)\left(q^{1 / 2}+\lambda^{1 / 2}\right) \\
& \left(-1+q^{1 / 2} \lambda^{1 / 2}\right)\left(-1+q^{3 / 2} \lambda^{1 / 2}\right)\left(-\left(q^{p 1}+q^{p 2}\right)\left(q^{1 / 2}+\lambda^{1 / 2}\right)\right. \\
& \left(-1+q^{1 / 2} \lambda^{1 / 2}\right)\left(1-q+q^{3}+q^{1 / 2}\left(1+(-1+q) q^{2}\right) \lambda^{1 / 2}\right) \\
& +q(q+1)(-1+q)^{2} q^{-(5 / 2)+p 1+p 2}\left(1+q^{1 / 2} \lambda^{1 / 2}\right) \\
& \left.\left.\left(-1+q\left(1-q+(-1+q) q^{1 / 2} \lambda^{1 / 2}+q(1+(-1+q) q) \lambda\right)\right)\right)\right]
\end{aligned}
$$

$$
\begin{aligned}
g_{2 \lambda^{(1)}, \lambda^{(2)}}(q, \lambda)= & \frac{1}{2(-1+q)^{4} q(q+1)}\left[\lambda ^ { - 2 + p 1 + p 2 } \left(( - 1 ) ^ { p 1 + p 2 } ( q + 1 ) \left(-3(-1)^{p 1+p 2}\right.\right.\right. \\
& q\left(q^{1 / 2}+\lambda^{1 / 2}\right)^{4}\left(-1+q^{1 / 2} \lambda^{1 / 2}\right)^{4}+(-1+q)^{4}(q+\lambda)(-1+q \lambda) \\
& \left.\left(-1+\lambda^{2}\right)\right)+\left(q^{1 / 2}+\lambda^{1 / 2}\right)^{2}\left(-1+q^{1 / 2} \lambda^{1 / 2}\right)^{2}(-(q+1) \\
& \left(-1-(-5+q) q-3(-1+q) q^{1 / 2} \lambda^{1 / 2}+(1+(-5+q) q) \lambda\right) \\
& \left(-1+q-q^{2}+(-1+q) q^{1 / 2} \lambda^{1 / 2}+(1+(-1+q) q) \lambda\right) \\
& +2 q(-1+\lambda)\left(q^{1 / 2-p 2}\left(q^{3 / 2}+\lambda^{1 / 2}\right)\left(-1+q^{1 / 2} \lambda^{1 / 2}\right)\right. \\
& \left.\left.+q^{p 1}\left(-q+q^{1 / 2}\left(-1+q^{2}\right) \lambda^{1 / 2}+q^{2} \lambda\right)\right)\right)+2 q^{-(3 / 2)-p 2} \\
& \left(q^{1 / 2}+\lambda^{1 / 2}\right)\left(-1+q^{1 / 2} \lambda^{1 / 2}\right)\left(-q(q+1)(-1+q)^{2} q^{p 1}\right. \\
& \left(q^{1 / 2}-\lambda^{1 / 2}\right)\left(-1+\lambda^{1 / 2}\right)\left(1+\lambda^{1 / 2}\right)\left(q^{3 / 2}+\lambda^{1 / 2}\right)\left(1+q^{1 / 2} \lambda^{1 / 2}\right) \\
& \left(-1+q^{3 / 2} \lambda^{1 / 2}\right)-q^{3 / 2}\left(q^{1 / 2}+\lambda^{1 / 2}\right)\left(-1+q^{1 / 2} \lambda^{1 / 2}\right)(-1+\lambda) \\
& \left(-q^{2}+q^{4}-q^{5}+\lambda^{1 / 2}\left(-q^{1 / 2}+q^{3 / 2}-q^{7 / 2}+q^{9 / 2}\right)+\lambda\left(1-q+q^{3}\right)\right. \\
& +q^{p 1+p 2}\left(-1+q-q^{3}+q^{1 / 2}\left(-1+q-q^{3}+q^{4}\right) \lambda^{1 / 2}\right. \\
& \left.\left.\left.\left.+q^{2}\left(1+(-1+q) q^{2}\right) \lambda\right)\right)\right)\right] \\
&
\end{aligned}
$$$$
g_{\lambda^{(2)}, \lambda^{(2)}}(q, \lambda)=\frac{1}{2(-1+q)^{4}(1+q)}\left[q ^ { - ( 9 / 2 ) - p 1 - p 2 } \lambda ^ { - 2 + p 1 + p 2 } \left(2(-1+q)^{2} q^{2}(1+q)\right.\right.
$$ 


$$
\begin{aligned}
& \left(q^{1 / 2}-\lambda^{1 / 2}\right)\left(-1+\lambda^{1 / 2}\right)\left(1+\lambda^{1 / 2}\right)\left(q^{1 / 2}+\lambda^{1 / 2}\right)\left(q^{3 / 2}+\lambda^{1 / 2}\right) \\
& \left(-1+q^{1 / 2} \lambda^{1 / 2}\right)\left(q^{2}-q^{3}+q^{4}+q^{3 / 2+p 1}\left(q^{1 / 2}+\lambda^{1 / 2}\right)\left(-1+q^{1 / 2} \lambda^{1 / 2}\right)\right. \\
& \left.+q^{3 / 2} \lambda^{1 / 2}-q^{5 / 2} \lambda^{1 / 2}-\lambda+q \lambda-q^{2} \lambda\right)+q^{p 2}(1+q)\left(2(-1+q)^{2}\right. \\
& q^{7 / 2}\left(q^{1 / 2}+\lambda^{1 / 2}\right)^{2}\left(-1+q^{1 / 2} \lambda^{1 / 2}\right)^{2}\left(q^{2}-(-1+q) q^{1 / 2} \lambda^{1 / 2}-\lambda\right) \\
& (-1+\lambda)-q^{7 / 2+p 1}\left(-1+q^{1 / 2} \lambda^{1 / 2}\right)\left(3(-1)^{2(p 1+p 2)} q\left(q^{1 / 2}+\lambda^{1 / 2}\right)^{4}\right. \\
& \left(-1+q^{1 / 2} \lambda^{1 / 2}\right)^{3}+\left(q^{1 / 2}+\lambda^{1 / 2}\right)^{2}\left(-1+q^{1 / 2} \lambda^{1 / 2}\right)(-1-(-5+q) q \\
& \left.-3(-1+q) q^{1 / 2} \lambda^{1 / 2}+(1+(-5+q) q) \lambda\right)\left(-1+q-q^{2}+(-1+q)\right. \\
& \left.q^{1 / 2} \lambda^{1 / 2}+(1+(-1+q) q) \lambda\right)+(-1)^{p 1+p 2}(-1+q)^{4}\left(1+q^{1 / 2} \lambda^{1 / 2}\right) \\
& \left.\left.\left.\left.(q+\lambda)\left(-1+\lambda^{2}\right)\right)\right)\right)\right]
\end{aligned}
$$

\section{Connected sum of Trefoil and Hopf link with framing $p_{1}$ on Trefoil and fram-} ings $p_{1}$ and $p_{2}$ on the hopf link

$$
\begin{aligned}
& g_{\lambda^{(1)}, \lambda^{(1)}}(q, \lambda)=q^{-3 / 2}\left[( - 1 ) ^ { p _ { 1 } + p _ { 2 } } ( - 1 + \lambda ) \lambda ^ { ( p _ { 1 } + p _ { 2 } ) / 2 } \left(-\left(q^{1 / 2}\left(1+q^{2}\right)\right)\right.\right. \\
& +\left(-1+q^{3}\right) \lambda^{1 / 2}+q^{1 / 2}\left(1+q+q^{2}\right) \lambda-\left(-1+q^{3}\right) \lambda^{3 / 2} \\
& \left.\left.-q^{3 / 2} \lambda^{2}+(-1+q) q \lambda^{5 / 2}\right)\right] \\
& g_{2 \lambda^{(1)}, \lambda^{(1)}}(q, \lambda)=\frac{-1}{(-1+q) q^{3}}\left[(-1)^{p_{2}}\left(-1+\lambda^{1 / 2}\right)\left(1+\lambda^{1 / 2}\right)\left(q^{1 / 2}+\lambda^{1 / 2}\right)\right. \\
& \left(-1+q^{1 / 2} \lambda^{1 / 2}\right) \lambda^{\left(1+2 p_{1}+p_{2}\right) / 2}\left(q ^ { 1 / 2 } \left[\left(-1+\left(q^{1 / 2}-\lambda^{1 / 2}\right)\right.\right.\right. \\
& \left.\left.\left(q^{1 / 2}(q(-1+\lambda)-\lambda)-\lambda^{1 / 2}\right)\right)\right]^{2}\left(q^{1 / 2}+\lambda^{1 / 2}\right)\left(-1+q^{1 / 2} \lambda^{1 / 2}\right) \\
& +q^{p_{1}}\left(1+q^{3}+q^{4}+q^{6}+q^{1 / 2}\left(1-q^{4}\left(-1+q+q^{3}\right)\right) \lambda^{1 / 2}\right. \\
& -q\left(1+q+2 q^{3}+q^{4}+q^{5}+q^{6}+q^{7}\right) \lambda \\
& +q^{3 / 2}\left(-1-q+q^{7}+q^{8}\right) \lambda^{3 / 2}+q^{3}\left(1+q^{2}+2 q^{3}+q^{6}\right) \lambda^{2} \\
& +q^{7 / 2}\left(1+q^{3}-q^{4}+q^{6}-2 q^{7}\right) \lambda^{5 / 2}+q^{8}(-1+(-1+q) q) \lambda^{3} \\
& \left.\left.\left.+q^{15 / 2}\left(-1+q-q^{3}+q^{4}\right) \lambda^{7 / 2}-(-1+q)^{2} q^{8}(1+q) \lambda^{4}\right)\right)\right](B \\
& g_{\lambda^{(2)}, \lambda^{(1)}}(q, \lambda)=\frac{-1}{-1+q}\left[(-1)^{p_{2}} q^{-17 / 2-p_{1}}\left(q^{1 / 2}+\lambda^{1 / 2}\right)\left(-1+q^{1 / 2} \lambda^{1 / 2}\right)(-1+\lambda)\right. \\
& \lambda^{\left(1+2 p_{1}+p_{2}\right) / 2}\left(q^{11 / 2}+q^{15 / 2}+q^{17 / 2}+q^{23 / 2}+q^{6+p_{1}}\right. \\
& \left(-1+\left(q^{1 / 2}-\lambda^{1 / 2}\right)\left(q^{1 / 2}(q(-1+\lambda)-\lambda)-\lambda^{1 / 2}\right)\right)^{2}
\end{aligned}
$$




$$
\begin{aligned}
& \left(q^{1 / 2}+\lambda^{1 / 2}\right)\left(-1+q^{1 / 2} \lambda^{1 / 2}\right)+\lambda^{1 / 2}\left(q^{4}+q^{6}-q^{7}-q^{11}\right) \\
& +\lambda\left(-q^{7 / 2}-q^{9 / 2}-q^{11 / 2}-q^{13 / 2}-2 q^{15 / 2}-q^{19 / 2}-q^{21 / 2}\right) \\
& +\lambda^{3 / 2}\left(-q^{2}-q^{3}+q^{9}+q^{10}\right)+\lambda^{2}\left(q^{5 / 2}+2 q^{11 / 2}+q^{13 / 2}+q^{17 / 2}\right) \\
& +\lambda^{5 / 2}\left(2 q-q^{2}+q^{4}-q^{5}-q^{8}\right)+\lambda^{3}\left(q^{3 / 2}-q^{5 / 2}-q^{7 / 2}\right) \\
& \left.\left.+\lambda^{7 / 2}\left(-1+q-q^{3}+q^{4}\right)+\lambda^{4}\left(-q^{1 / 2}+q^{3 / 2}+q^{5 / 2}-q^{7 / 2}\right)\right)\right](\mathrm{B} .
\end{aligned}
$$

\section{References}

[1] E. Witten, "Chern-Simons Gauge Theory as a String Theory," hep-th/9207094

[2] R. Gopakumar, C. Vafa, "M-Theory and Topological Strings, I," hep-th/9809187.

[3] R. Gopakumar, C. Vafa, "On the Gauge Theory/ Geometry Correspondence," hep-th/9811131.

[4] R. Gopakumar, C. Vafa, "M-Theory and Topological Strings, II," hep-th/9812127.

[5] H. Ooguri, C. Vafa, "Knot Invariants and Topological Strings," Nucl. Phys. B577, 419, (2000), hep-th/9912123.

[6] J. M. F. Labastida, M. Marino, "Polynomial Invariants for Torus Knots and Topological Strings," hep-th/0004196.

[7] P. Ramadevi, T. Sarkar, "On Link Invariants and Topological String Amplitudes," Nucl. Phys. B 600 (2001) 487.

[8] J. M. F Labastida, M. Marino, C. Vafa, "Knots, Links and Branes at Large N," JHEP11 (2000) 007, hep-th/0010102

[9] M. Marino, C. Vafa, "Framed Knots at Large $N$," hep-th/0108064.

[10] Pravina Borhade, P. Ramadevi, Tapobrata Sarkar, "U(N) Framed Links, Three-Manifold Invariants, and Topological Strings," Nucl.Phys. B678 (2004) 656-681.

[11] G. 't Hooft, "A Planar Diagram Theory for Strong Interactions," Nucl. Phys. B 72 (1974) 461.

[12] M. Marino, "Chern-Simons theory, matrix integrals and perturbative three-manifold invariants," Commun.Math.Phys. 253 (2004) 25-49. 
[13] R. Dijkgraaf and C. Vafa, "Matrix models, topological strings and supersymmetric gauge theories," Nucl. Phys. B 6443 (2002).

[14] M. Aganagic, A. Klemm, M. Marino and C. Vafa, "Matrix model as a mirror of ChernSimons theory," JHEP 0402 (2004) 010.

[15] S. Sinha, C. Vafa, "SO and Sp Chern-Simons at Large N," hep-th/0012136.

[16] V. Bouchard, B. Florea, M. Marino, "Counting Higher Genus Curves with Crosscaps in Calabi-Yau Orientifolds," JHEP 0412(2004) 035, hep-th/0405083

[17] V. Bouchard, B. Florea, M. Marino, "Topological Open String Amplitudes On Orientifolds," JHEP 0502 (2005) 002, hep-th/0411227.

[18] J.M.F. Labastida, M. Marino, " A New Point of View in the Theory of Knot and Link Invariants," math.QA/0104180, J. Knot Theory Ramifications 11 (2002) 173. 